Check for updates

Cite this: RSC Adv., 2022, 12, 7635

Received 26th January 2022

Accepted 2nd March 2022

DOI: 10.1039/d2ra00566b

rsc.li/rsc-advances

\section{Recent progress in the applications of gold-based nanoparticles towards tumor-targeted imaging and therapy}

\author{
Xinxin Li,,$^{\text {ac }}$ Yiwei Zhang, $\dagger^{\text {ac }}$ GuangKuo Liu, ac ${ }^{\text {ac }}$ Zil luo, ${ }^{c}$ Lu Zhou, ${ }^{d}$ Yanan Xue*a \\ and Min Liu (DD*bc
}

\begin{abstract}
Cancer death rate remains high all over the world, scientists are paying increasing attention to meet the requirements for precise diagnosis and therapy. Therefore, early diagnosis and active treatment can effectively improve the five-year survival rate of patients. In recent years, gold-based nanomaterials have received increasing attention in medical fields due to their excellent biocompatibility, low toxicity and unique properties. In addition, because of the inherent nature of gold nanomaterials including for computed tomography (CT), fluorescence/optical imaging (FI/OI), surface enhanced Raman spectroscopy imaging (SERS), photoacoustic imaging (PAI) and photothermal therapy (PTT), various gold nanomaterials were developed as theranostic nanoplatforms. In this review, we summarized the latest developments of nanomaterials in imaging and combined therapy, and the prospects for the future application of gold-based theranostic nanoplatforms were also proposed.
\end{abstract}

\section{Introduction}

In 2022, the United States estimates that there will be 1918030 new cancer cases and 609360 cancer deaths. The number of cancer deaths was increasing every year until 1991, after which the mortality rate has declined year by year. By 2017, the estimated number of cancer-caused deaths was 2.9 million. ${ }^{\mathbf{1 , 2}}$ Early detection, early diagnosis and early treatment was the only way of improving the survival rate for cancer patients.

Gold nanomaterials have been widely used in biomedical research due to their unique properties and good biocompatibility. ${ }^{3-6}$ As early as the 1970 s, colloidal gold had been used in the treatment of rheumatoid arthritis patients. Thereafter, gold nanoparticles $(27 \mathrm{~nm})$ were used as carriers to load recombinant human tumor necrosis factor for treating advanced malignant tumor patients and then progressed to phase I clinical research. Results of clinical experiment showed that no obvious side effects were observed in the patients. ${ }^{7}$ In another clinical study registered in Russia, a $15 \mathrm{~nm}$ gold shell was used for arterial porridge in the photothermal treatment group,

\footnotetext{
${ }^{a}$ Hubei Key Laboratory for Novel Reactor and Green Chemistry Technology, School of Chemical Engineering and Pharmacy, Wuhan Institute of Technology, Wuhan 430205, China

${ }^{b}$ Key Laboratory of Optoelectronic Chemical Materials and Devices of Ministry of Education, Jianghan University, Wuhan 430056, China

'Institute for Interdisciplinary Research, Jianghan University, Wuhan 430056, China. E-mail:mliu2010@sinano.ac.cn

${ }^{d}$ Department of Medical Mycology, Shanghai Dermatology Hospital Affiliated to Tongji University, Shanghai 200443, China

$\dagger$ These authors controlled equally.
}

which achieved better clinical effect. ${ }^{8}$ These results demonstrated that gold nanomaterials have high scientific value and excellent clinical application prospects.

The synthesis and modification methods of gold nanoparticles (AuNPs) are mature, simple, and the structure is relatively easy to control (Scheme 1). Various AuNPs with different size and shape are prepared, such as spheres, ${ }^{\mathbf{9 , 1 0}}$ rods, ${ }^{11,12}$ clusters, ${ }^{13-16}$ cages, ${ }^{17,18}$ stars $^{18,19}$ and so on. Moreover, AuNPs possess some unique properties: (1) imaging, including computed tomography (CT), fluorescence/optical imaging, surface enhanced Raman spectroscopy imaging (SERS), photoacoustic imaging (PAI). (2) Therapy, including drug/gene delivery, photothermal therapy (PTT). On the basis of these properties, researchers continuously develop new methods for preparing/ modifying AuNPs so as to improve or obtain new performances. In this review, we summarized the recent development of gold-based nanoplatform including polymer-coated gold nanoplatform, gold-based hybrid nanoplatform, and membrane-coated gold nanoplatform, and their applications in tumor diagnosis and therapy were also classified and discussed. Moreover, the prospect for the development of gold-based nanoplatform is also proposed.

\section{Preparation and properties of gold nanoparticles}

AuNPs possess a variety of different sizes and shape, including sphere, rod, cage, cluster and so on. As time goes on, researchers can synthesize AuNPs with almost all sizes and shapes. There are 

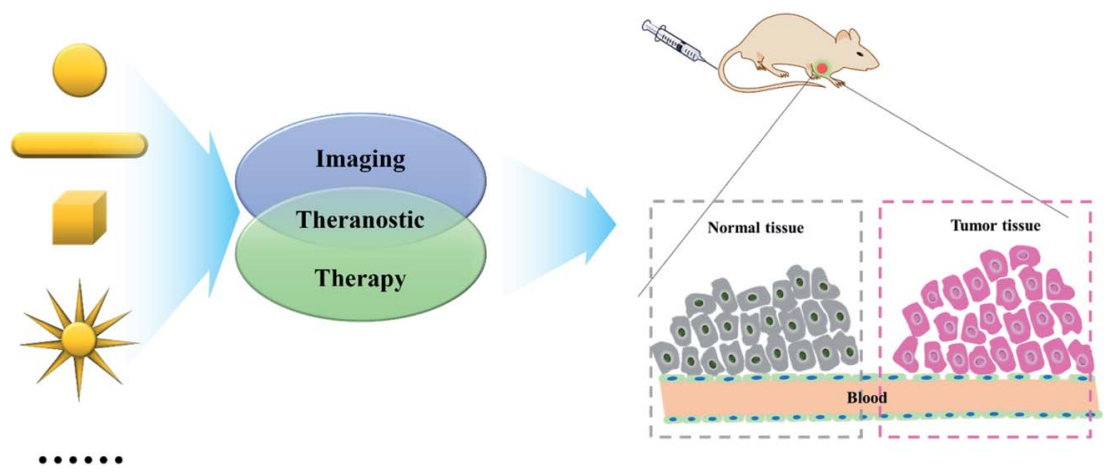

Scheme 1 Illustration of various shape of gold nanoparticles and their applications.

three main synthetic methods: (1) citric acid reduction $\mathrm{HAuCl}_{4}{ }^{20,21}$ Classic method for preparing gold nanosphere is citric acid reduction $\mathrm{HAuCl}_{4}$. The diameter of the gold nanospheres can be controlled at $15-150 \mathrm{~nm}$ via changing the concentration of sodium citrate, and diameter greater than $25 \mathrm{~nm}$ possess gold photothermal effect. (2) Seed-mediated method. ${ }^{22}$ Classic methods for preparing gold nanorod is seed-mediated method. Electrochemical methods and photochemical reduction are also used to prepare gold nanorod. Different from gold nanosphere, gold nanorods are anisotropic and have two absorption peaks of localized surface plasmon resonance (LSPR). The lateral LSPR band is fixed at approximately $520 \mathrm{~nm}$, while the longitudinal LSPR band is adjustable in the visible to near infrared (NIR) range. (3) Template method. ${ }^{23}$ Classic methods for preparing gold nanocage are template method which uses silver nanocube as a template and reduces the seed crystal to induce the growth of gold spheres around the gold template, thereby forming a mesoporous shell. The gold nanocage has a hollow and porous wall structure, which can be used as carrier.

Different kinds of AuNPs have various size and shape, however, these AuNPs usually possess some common properties. For example, due to the high atomic number and electron density, AuNPs can effectively absorb X-ray irradiation, which is superior to the iodine-based contrast agents used in clinic. Next, the absorption on AuNPs or other metal surfaces can enhance the intensity of the vibration spectra of Raman active molecules, which then developed into a new technology-surface enhanced Raman spectroscopy imaging (SERS) for detecting the margin of tumor. In addition, the irradiation of AuNPs with laser of certain wavelength induces localized temperature increases. On the one hand, the increased temperature will result in photothermal ablation, which is then widely used in tumor therapy; on the other hand, the heating will also cause a sound wave, which can be captured and transferred as photoacoustic imaging. AuNPs have many other properties, this review will then summarize and discuss in the following text.

\section{Polymer-coated gold nanoparticles for imaging and therapy}

When a variety of NPs enter the human body, the stability of these NPs is the key problem that needs to be addressed.
Polymer coating is a common technique. Meade group reported a strategy for early detection of pancreatic adenocarcinoma through conjugating dithiolane-Gd(III) complex to AuNPs (Fig. 1A) ${ }^{24}$ In this work, Gd-DO3A-C6 amine and Gd-DTPA-C4 amine were conjugated with lipoic acid followed by anchoring onto the surface of AuNPs. The results demonstrated that these conjugates could acquire MR images at both low and high magnetic field strengths due to the high Gd(III) payload, and could be used as potential candidates for diagnosis and treatment of pancreatic disease. Similarly, Zhang group fabricated c(RGDyC), Gd- and 99 mTc-labeled AuNPs (RGD@AuNPs-Gd99 $\mathrm{mTc})$ probe with different sizes of AuNPs (29, 51 and $80 \mathrm{~nm}$ ), and their potential radiosensitization therapy guided by MRI/ SPECT both in vitro and in vivo were also evaluated (Fig. 1B). ${ }^{25}$ The results demonstrated that the RGD@AuNPs-Gd99 mTc with $29 \mathrm{~nm}$ Au NPs were clearly the most efficient in vivo.

Undoubtedly, there simple decorations greatly improve the stability in vivo and endow them with excellent targeting ability. Along with going deep gradually into the research, these simple decorations cannot meet the demand. More and more multifunctional polymers are decorated onto the surface of AuNPs to endow them with more and better functions. Zhang group fabricated a multifunctional nanocomposite AuNP@CD-ADDOX/RGD via host-guest interaction for targeted cancer therapy ${ }^{26}$ In the work, AuNP@CD was firstly synthesized as the core to offer binding sites for adamantane-modified PEG ligands. Afterward, anticancer drug doxorubicin (DOX) was conjugated onto adamantane via hydrazine bond that could be broken under acid tumor environment (AD-Hyd-DOX), while RGD was conjugated onto adamantane via PEG ligand (ADPEG8-GRGDS). Thereafter, AD-Hyd-DOX and AD-PEG8-GRGDS were both anchored onto AuNP@CD via host-guest interaction. The results illustrated that AuNP@CD-AD-DOX/RGD could be uptaken by cancer cells and released DOX rapidly in the acid environment and thereby inducing cell apoptosis. A similar structure was also reported by Xia group. ${ }^{27}$ They reported a three-in-one nanoplatform for sensing, self-assembly and cascade catalysis via cyclodextrin modified AuNPs (CD@AuNPs). AuNPs were employed as fluorescent sensing based on guest replacement reaction, while the cyclodextrin on the surface of AuNPs not only endowed the AuNPs with the ability of self-assembly to form one- and two-dimensional architectures using tetrakis(4-carboxyphenyl) porphyrin as 

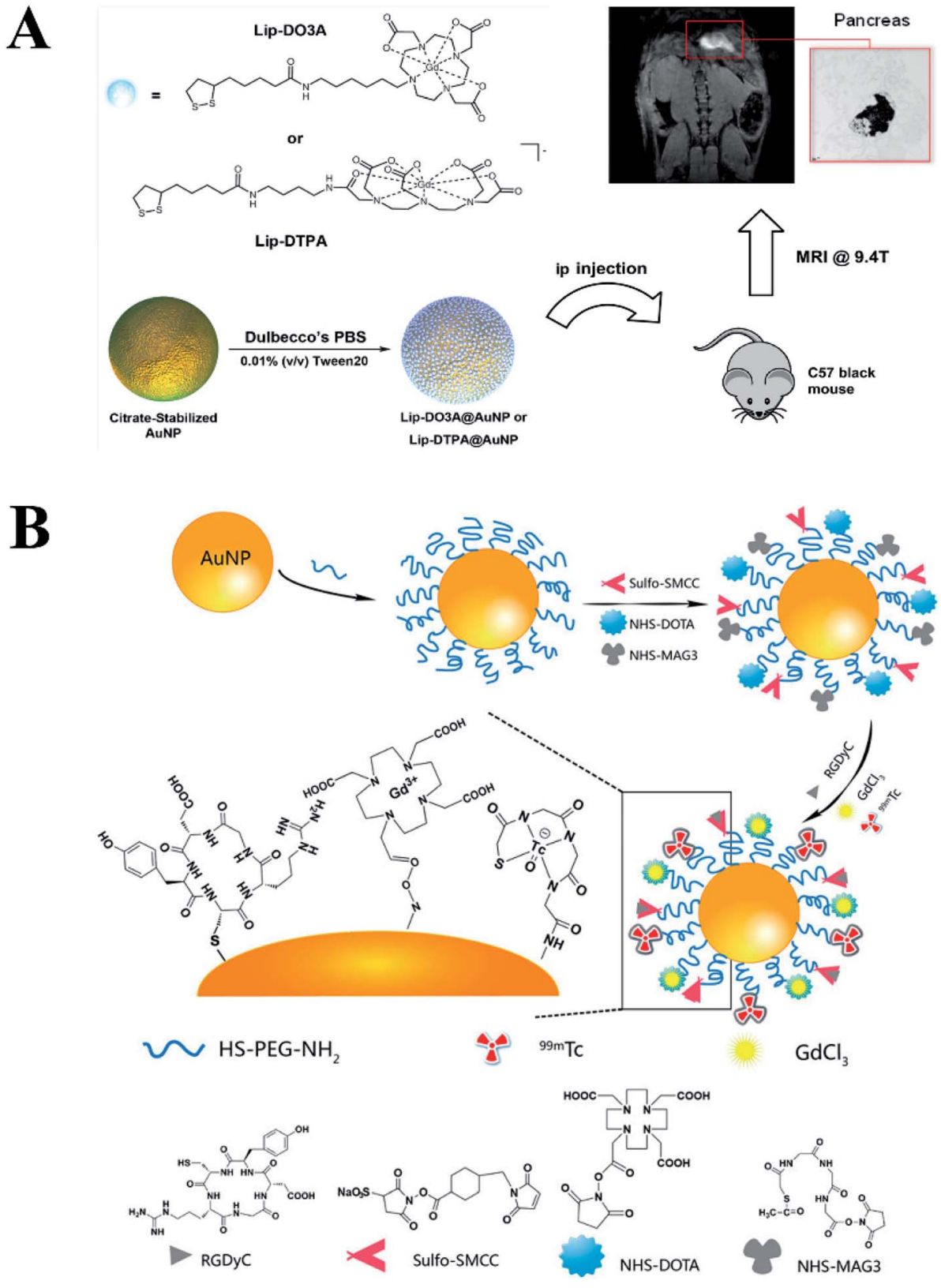

Fig. 1 (A) Gd(II) Chelates (Lip-DO3A and Lip-DTPA) were conjugated to the surface of AuNPs to create Lip-DO3A@AuNP and Lip-DTPA@AuNP. Reproduced with permission from ref. 24, copyright: 2016, American Chemical Society. (B) Schematic diagram of the procedure for fabrication of the RGD@AuNPs-Gd99 mTc Probes. Reproduced with permission from ref. 25, copyright: 2016, American Chemical Society.

mediator, but also endowed the AuNPs with the ability of catalytic activity.

Dendrimer is a category of nano-sized polymer with a welldefined composition and branched tree-like structure. The uniform size and globular shape make dendrimer a nanoplatform to encapsulate or stabilize various inorganic nanoparticles, including metal or metal derivatives. Shi group reported a facile approach to fabricate low generation poly(amidoamine) (PAMAM) dendrimer stabilized gold nanoparticles (Au DSNPs) for in vivo computed tomography (CT) imaging applications. ${ }^{28}$ In this work, PAMAM dendrimers (G2) were employed as stabilizers to form $\mathrm{Au}$ DSNPs through a simple hydrothermal method, followed by neutralizing with acetic anhydride. The as-prepared Au DSNPs with an Au core size of $5.6 \mathrm{~nm}$ performed much better in CT imaging of some major organs of rat than that of Omnipaque (a clinical contrast agent). Kono group modified PEG-PAMAM dendrons onto the surface of a gold nanorod (GNR) by adding PEG-PAMAM dendrimers (G2-G4) with a cystamine core during the GNR growing reaction..$^{29}$ The dendrimer modified GNR exhibited excellent biocompatibility against HeLa cells, however, the GNR-cored PEG-PAMAM dendrimers killed the HeLa cells effectively under near-infrared laser irradiation. In vivo experiments also demonstrated that the tumor volume obviously decreased with 
the irradiation of near infrared laser. Thereafter, Kono group further fabricated $\mathrm{pH}$-sensitive drug-dendrimer conjugatedhybridized gold nanorod for combined cancer photothermalchemotherapy (Fig. 2). ${ }^{30}$ Briefly, poly(ethylene glycol)-modified PAMAM G4 dendrimer (PEG-PAMAM) were covalently linked on the surface of mercaptohexadecanoic acid-functionalized gold nanorod (MHA-AuNR) followed by conjugation of anticancer drug doxorubicin (DOX) through acid-labile-hydrazone bond. The obtained PEG-DOX-PAMAM-AuNR particles could realize controlled release at different $\mathrm{pH}$ value. In addition, PEG-DOX-PAMAM-AuNR with irradiation groups showed significant tumor growth suppression or delay in comparison with the control groups, which illustrated that the combined photothermal-chemotherapy exhibited higher therapeutic efficacy then the single treatment.

Stem cell therapy is to transplant healthy stem cells into patients, thereby repairing damaged tissues, so as to achieve the purpose of treatment. How to effectively trace the trajectories of transplanted cells in vivo and understand the migration, homing and survival of transplanted cells remains a great challenge. Modo group reported an AuNP conjugate to track the transplanted stem cells (Fig. 3A). ${ }^{31}$ Briefly, Gd chelates were conjugated onto $\mathrm{Cy} 3$ labelled deoxythymidine oligonucleotides via click chemistry (Cy3-labeled Gd-DNA). Afterward, Cy3labeled Gd-DNA was anchored onto the surface of AuNPs through gold-thiol chemistry (DNA-Gd@AuNP). The diameter of the NPs increased from $15.3 \pm 1.4 \mathrm{~nm}$ (size of AuNPs observed by TEM) to $35 \pm 0.7 \mathrm{~nm}$ (hydrodynamic radius of DNAGd@AuNPs). The experiment results showed that DNAGd@AuNP exhibited remarkably $T_{1}$ relaxivity and could be used for in vivo detection of transplanted cells. The postmortem histological verification of DNA-Gd@AuNP in the transplanted cells further corroborated the results with that of in vivo MR images. DNA-Gd@AuNP therefore offer the opportunity to visualize the transplanted cells through MRI technique as well as reflect the information of the survival status and
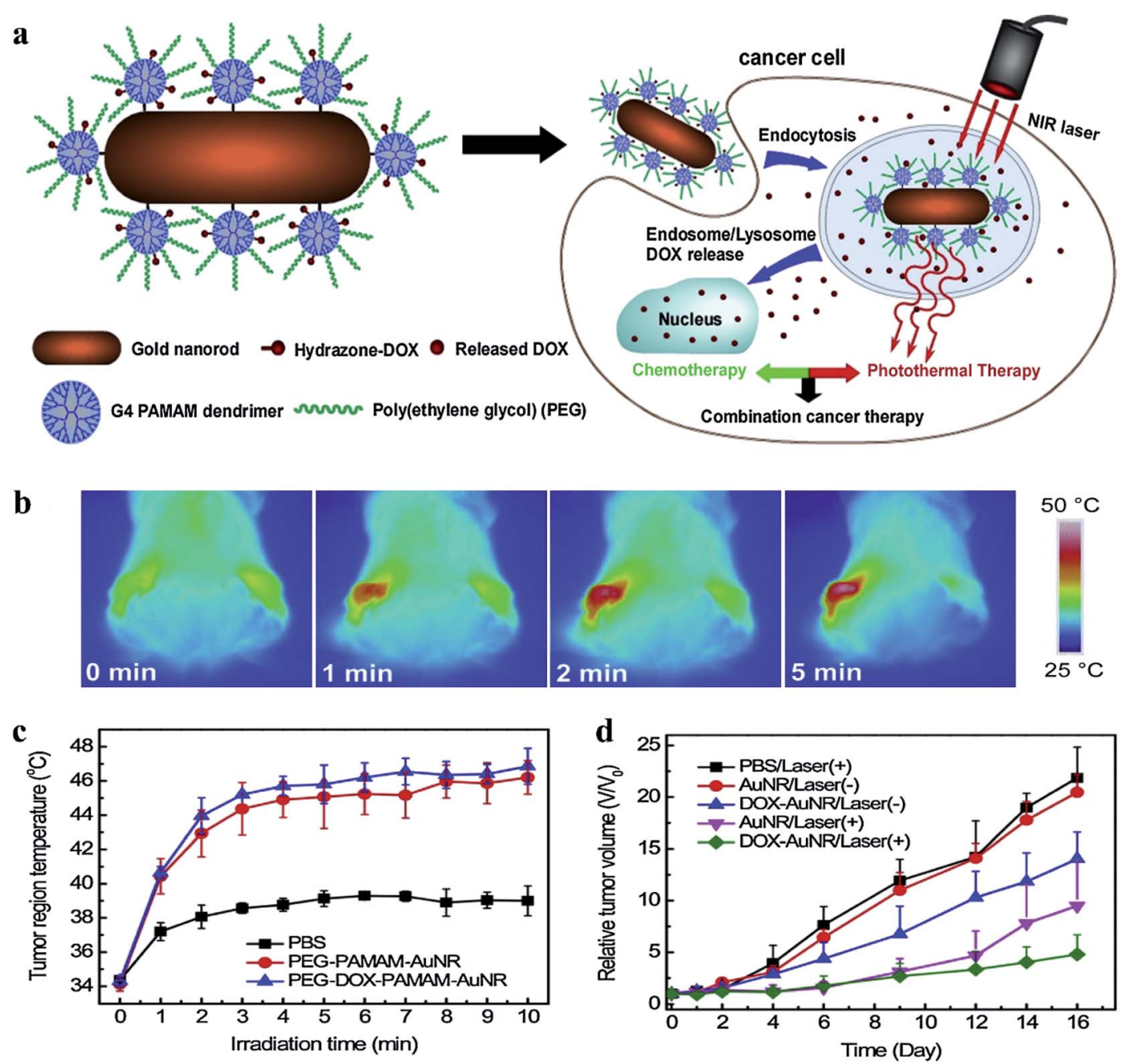

Fig. 2 (a) Schematic illustration of pH-sensitive PEGylated PAMAM dendrimer-doxorubicin conjugate-hybridized gold nanorod (PEG-DOXPAMAM-AuNR) for combined photothermal-chemotherapy. (b) Thermographs of PEG-DOX-PAMAM-AuNR-injected mouse receiving photothermal treatment for different periods of time. (c) Time courses of temperature variation in the left tumor region under NIR laser irradiation. (d) Tumor growth profiles of tumor-bearing mice after intravenous injection of samples. Reproduced with permission from ref. 30, copyright: 2014, ScienceDirect. 


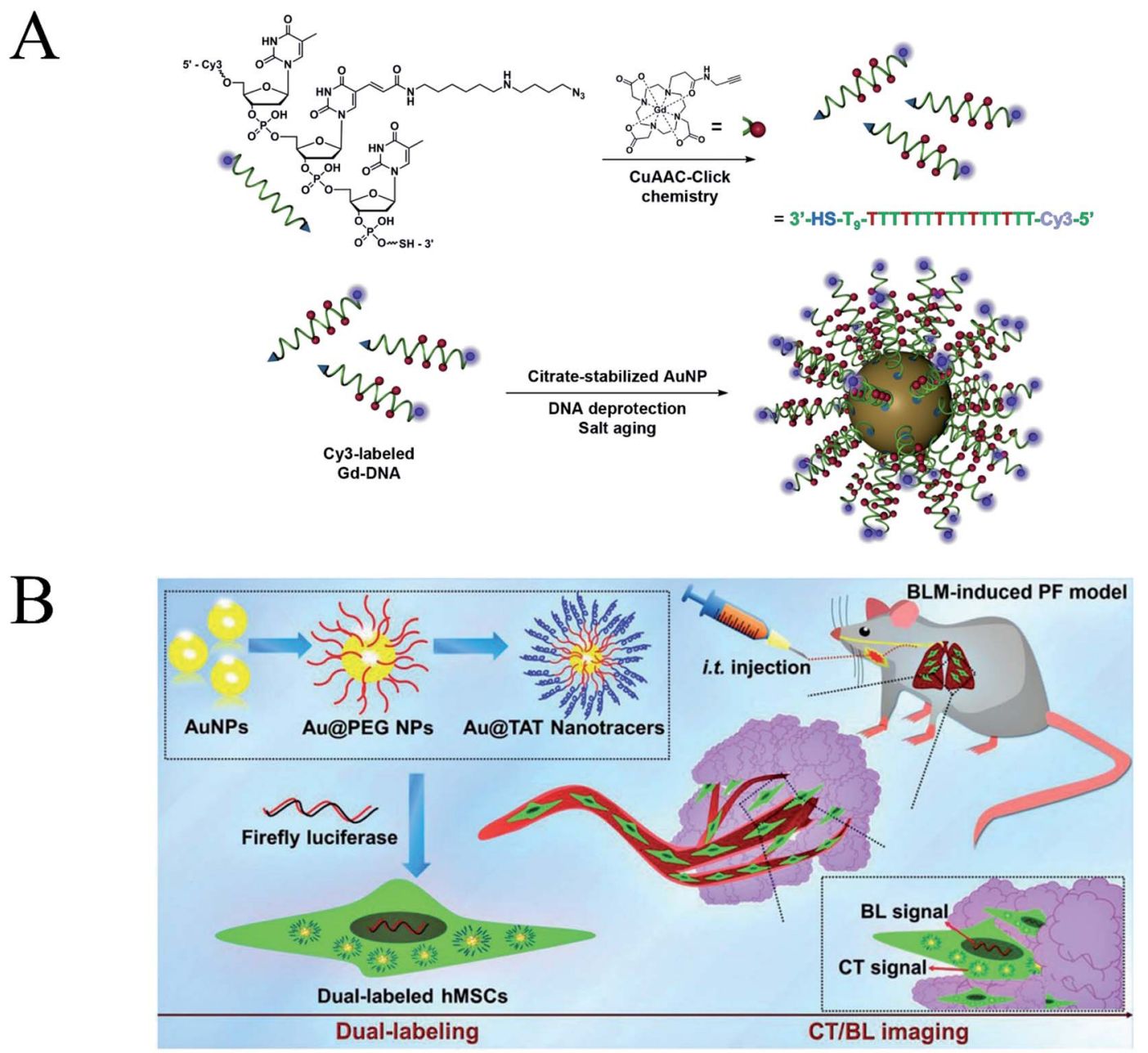

Fig. 3 (A) DNA-Gd@AuNP synthesis and stability. Particles consist of a gold nanoparticle core loaded with DNA to which the Gd-HPDO3A and Cy3 moieties are attached. Reproduced with permission from ref. 31, copyright: 2016, ScienceDirect. (B) Schematic illustration of the duallabeling CT/BLI strategy that combines the exogenous Au@TAT nanotracers and the endogenous RfLuc for tracking of the transplanted hMSCs via intratracheal (i.t.) administration in BLM-induced PF. Reproduced with permission from ref. 33, copyright: 2019, Wiley.

location of the transplanted cells, which help us understand the therapeutic mechanism. In addition, Drezek group fabricated AuNP-PAMAM conjugates as non-viral transfection agents, which was prepared by crosslinking PAMAM dendrimers to carboxylic-terminated AuNP through EDC chemistry. ${ }^{32}$ Thereafter, the optimal vector was obtained through adjusting the ratio of amine and carboxyl groups, and thereby yielding high transfection efficiency and low cytotoxicity. Zhang group modified AuNPs with red-emitting firefly luciferase (RfLuc)based bioluminescence (BL) tags for CT/BL bimodal imaging to track the transplanted cells in a murine model of idiopathic pulmonary fibrosis (IPF) (Fig. 3B). ${ }^{33}$ This technique combined $\mathrm{CT}$ and BL imaging enables in situ visualization of the location, distribution and survival of the transplanted cells.

The above-mentioned literature summarized the examples that various polymer/DNA chains were directly anchored onto the surface of AuNPs, which construct a relatively simple and easy system for diagnosis and therapy. Thereafter, some response mechanisms were also introduced into the modification of AuNPs to further improve the imaging or therapeutic effects. Shuai group loaded DOX into a pH-sensitive core and acted as a template to introduce a gold nanocage followed by conjugation of polyethylene glycol (PEG). ${ }^{34}$ This DOXloaded $\mathrm{pH}$-sensitive gold nanocage (D-PGNC) was relative stable due to the cross-linking and gold decoration of interlayer in the blood stream, but turned on in the tumor region due to the acid tumor microenvironment, high concentration of GSH and nearinfrared (NIR) irradiation. Afterward, the results of synergistic anticancer therapy in vivo illustrated that D-PGNC plus NIR groups showed the longest survival time (a 45 days survival rate of $83.3 \%$ ) than that of control groups.

Next, Klajn group developed a new concept to assemble the NPs mediated by light, which did not require the modification of light-responsive ligands. ${ }^{35}$ In their work, a photoswitchable medium was introduced, whose acidity could be modulated reversibly under visible light and then affected the NPs interaction. Therefore, these NPs could quantitatively assemble or disperse. Gao group developed smart responsive AuNP that 
$\mathbf{a}$

b
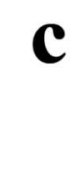

Diazirine-decorated NPS

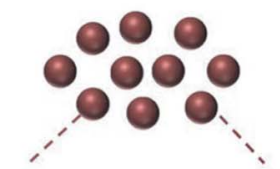

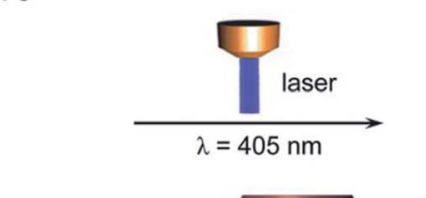
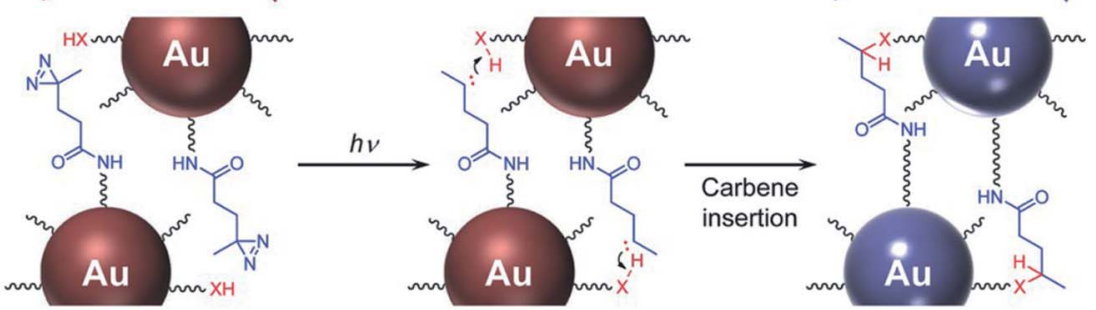

$\mathrm{X}=\mathrm{C}, \mathrm{N}, \mathrm{O}, \mathrm{S}$
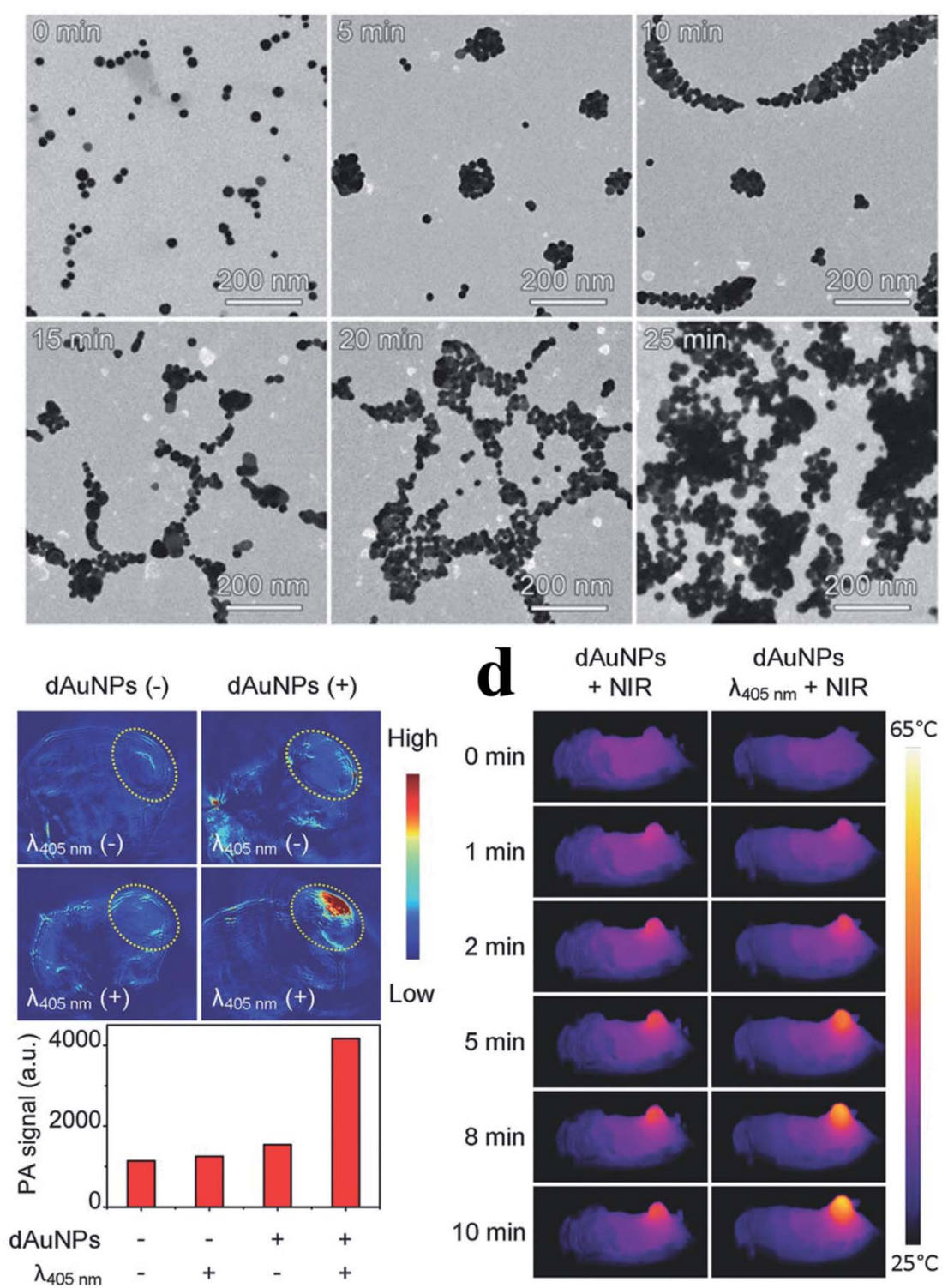

dAuNPs

dAuNPs

$\lambda_{405 \mathrm{~nm}}$

Fig. 4 (a) Schematic illustration of light-triggered assembly of dAuNPs. (b) TEM images before and after illuminated with $405 \mathrm{~nm}$ laser different periods of time. (c) PA images and quantified PA signal of the tumorous sites of mice receiving treatments with different combinations of $\lambda_{405 \mathrm{~nm}}$ laser irradiation and intravenous delivery of dAuNPs $\left(100 \mu \mathrm{L}, 2 \mathrm{mg} \mathrm{mL}^{-1}\right)$. (d) Photothermal images of tumor-bearing mice for showing the in vivo cross-linking effect of dAuNPs on tumor local temperature against irradiation time of $808 \mathrm{~nm}$ laser. Reproduced with permission from ref. 36 , copyright: 2017, Wiley. 
A

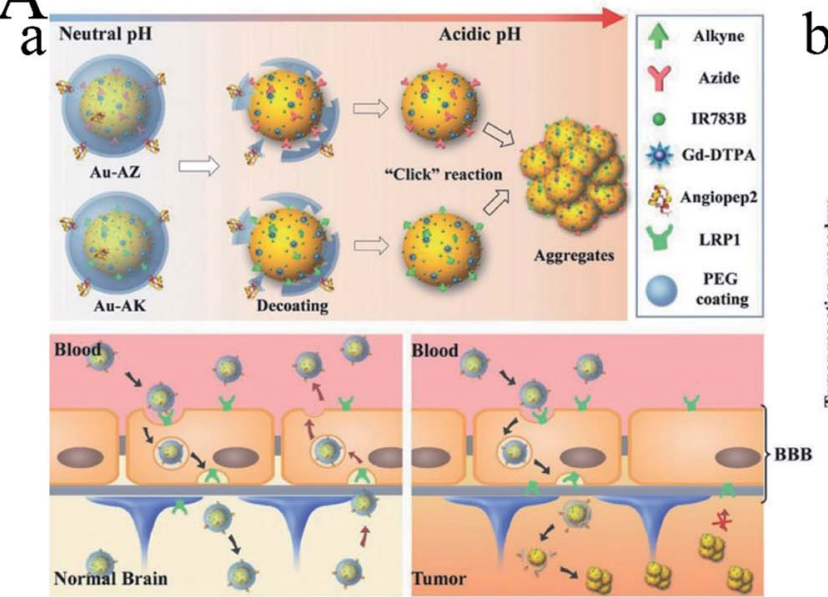

B

a
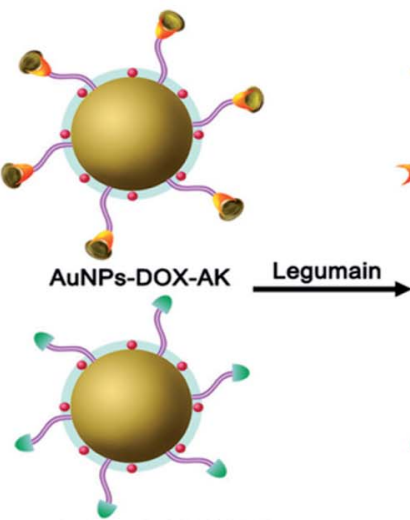

AuNPs-DOX-CABT

Click

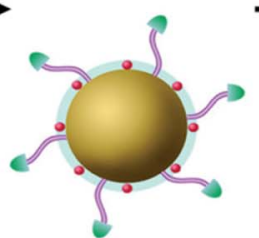

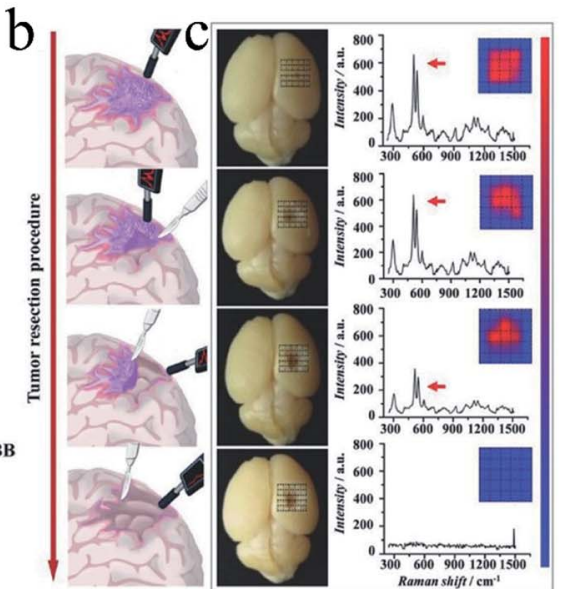
cycloaddition

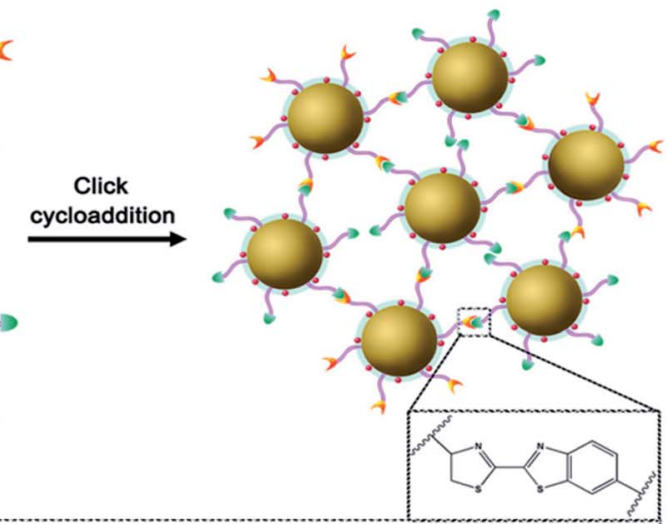

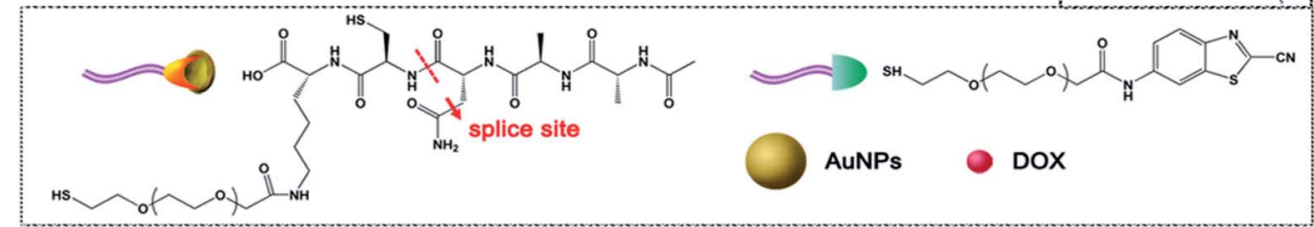

b
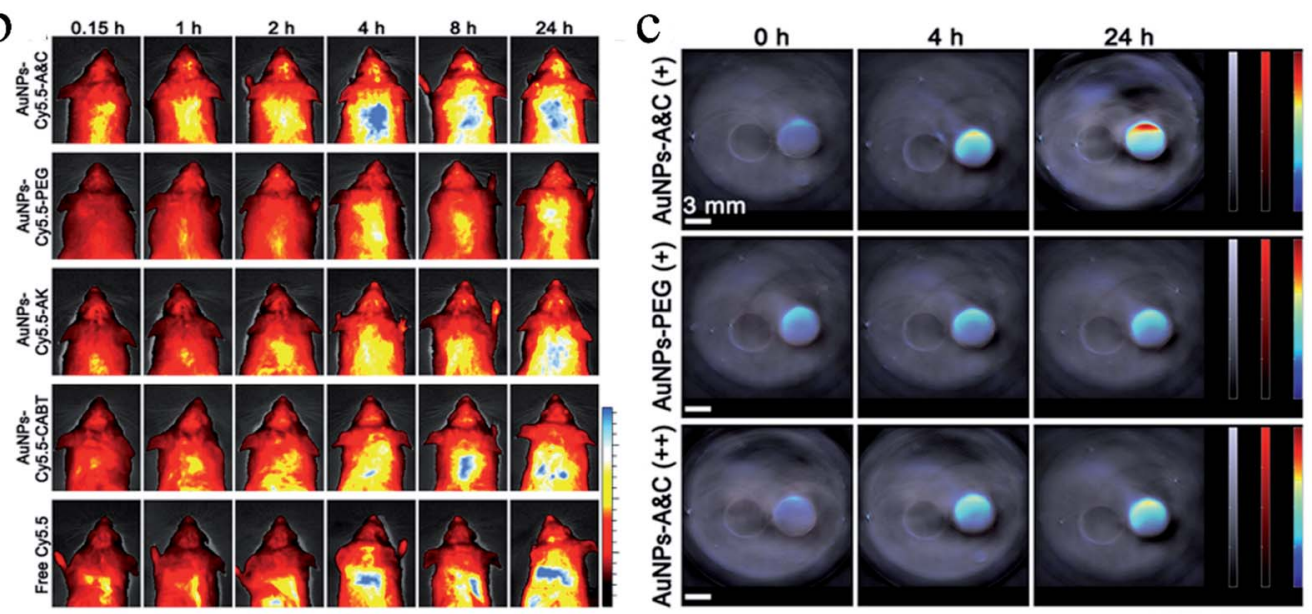

Fig. 5 (A) Guiding brain-tumor surgery by acid-responsive gold nanoprobes. (a) The cleavage of the PEG coating in physiological acidity triggers aggregation between Au-AZ and Au-AK via "click" cycloaddition reactions. (b) Au-AZ/Au-AK mixture intraoperatively guiding glioma resection using a handheld Raman detector. Cartoons illustrating the procedure of Raman-spectroscopy-guided glioma resection. (c) Photographs of the excised mouse brain bearing U87MG glioma xenografts and Raman spectra at the tumor site during the image-guided tumor resection. Surgery was conducted at $24 \mathrm{~h} \mathrm{PI}$ of the Au-AZ/Au-AK mixture ( $1: 1$ molar ratio, totaling $600 \mathrm{pmol} \mathrm{kg}{ }^{-1}$ ). The intensities of the characteristic Raman twin peak were quantified in a $5 \times 5$ grid covering the tumor cutting bed. Reproduced with permission from ref. 37, copyright: 2017, Wiley. (B) (a) Diagram depicting the legumain-triggered aggregation and composition of AuNPs-DOX-A\&C. The AuNPs-DOX-AK undergoes a cleavage by 
could covalently crosslinking in the tumor region under $405 \mathrm{~nm}$ laser irradiation, via a diazirine terminal group of $\mathrm{PEG}_{5000}$ ligands on the surface of AuNPs $(20.5 \mathrm{~nm}) .{ }^{36}$ As shown in Fig. 4, $\mathrm{NH}_{2}$ groups were introduced to the surface of AuNPs via PEG ligands followed by conjugating with NHS-diazirine (succinimidyl 4,4-azipentanoate) to obtain diazirine-decorated AuNPs (denoted as dAuNPs). Thereafter, the average size of the asprepared dAuNPs $(20.5 \pm 1.9 \mathrm{~nm})$ changed into largely forming particle aggregates (700 $\mathrm{nm}$ after $15 \mathrm{~min}$ of irradiation), and the size of aggregates gradually increased with the increase of the exposure time to $405 \mathrm{~nm}$ laser irradiation. In addition, in vivo experiment illustrated that $\mathrm{PA}$ signal of the tumor sites (Fig. 4c) obviously enhanced after $405 \mathrm{~nm}$ laser irradiation, and the photothermal images (Fig. 4d) of the tumor bearing mice further illustrated the in vivo crosslinking effect of dAuNPs in the tumor region after irradiation both at 405 and $808 \mathrm{~nm}$ laser. Meanwhile, the tumors holding cross-linked dAuNPs groups were significantly reduced in size with the $808 \mathrm{~nm}$ irradiation compared to the control groups, which also exhibited improved survival rate. In general, AuNPs with small size have longer blood retention times, but the small ones also possess low imaging capability and photothermal therapy. The mechanism of light-induced aggregation just makes up the limitation.

However, how to distinguish the tumor margins faced great challenges in surgery. Li group developed a pair of gold nanoprobes that could cross the blood-brain barrier (BBB) with the help of angiopep2 peptides (Fig. 5A). ${ }^{37}$ Thereafter, these gold nanoprobes could further assemble into aggregations via bioorthogonal reactions under the acidic tumor environment. As time went on, the aggregates continuously trapped into the tumor region and thereby enhancing both magnetic resonance (MR) signal and surface-enhanced resonance Raman spectroscopy (SERRS) signals. Therefore, the MR signal could be used for diagnosis, while SERRS signal could be used for intraoperatively guide tumor excision with the assistance of a handheld Raman scanner. These promising candidates could significantly improve the accuracy of brain-tumor surgery as well as the possible extension of other tumor resections. Gao group developed an aggregation mechanism triggered by legumain between Ala-Ala-Asn-CysLys modified AuNPs (AuNPs-AK) and 2-cyano-6aminobenzothiazole modified AuNPs (AuNPs-CABT) (Fig. 5B). ${ }^{38}$ The obtained AuNPs-A\&C could realize aggregation under certain condition both in vitro and in vivo. In vivo fluorescence imaging and PA imaging demonstrated that AuNPs-A\&C could selectively accumulate in the glioma site. After loading doxorubicin (DOX), the median survival time increased to $288 \%$ in comparison to the saline group. Therefore, the AuNPs-A\&C system could realize precisely diagnosis and therapy of glioma. Compared to the aggregation mediated by photo mentioned above, chemical reactions triggered aggregation could form more stable gold aggregates.

In this section, various kinds of polymer-coated AuNPs were reviewed and discussed. On the one hand, these decorations significantly improve the stability of AuNPs in vivo, thereby increasing the accumulation of AuNPs in the tumor region. On the other hand, these decorations also endow AuNPs with some new characters, including targeting ability, pH sensitivity, light response, which further expand the applications of AuNPs.

\section{Gold-based hybrid NPs for imaging and therapy}

Polymer coating can endow the AuNPs with lots of new properties, however, hybrid AuNPs with inorganic/organic/metal materials will open a new world and endow AuNPs with some characters, which polymer-coating cannot offer. Silica, a common inorganic material, is often used as surface coating, which can increase the inertness of NPs in vivo as well as endow the NPs with the ability of loading drug. Prabaharan group fabricated silica@gold NPs followed by conjugation of guar gum

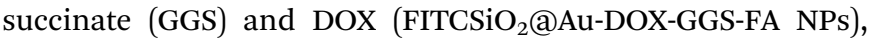
which was used of CT/fluorescence dual imaging and chem/ phototherapy. ${ }^{39}$ The obtained NPs exhibited fluorescence and Xray attenuation properties due to the $\mathrm{SiO}_{2} @ \mathrm{Au}$ hybrid nanostructure. In addition, DOX was conjugated onto the $\mathrm{SiO}_{2} @ \mathrm{Au}$ NPs via hydrazone bond that could be cleaved under acidic condition. Next, many other elements are developed.

Selenium nanoparticles and selenodiazole derivatives induce cell apoptosis by generating Compton effect and photoelectric effect, which can enhance X-ray-based cancer radiotherapy. However, the imaging capabilities of seleniumcontaining radiosensitizers are often limited by individual differences. AuNPs, as high-Z and extremely high-chemical inert materials, are widely used as radiosensitizers and contrast agents due to their large photoelectric absorption coefficient at the tumor site. Chen group developed a simple way to fabricate a ternary heterostructure radiosensitizer (SeAuFe-EpC) with core/satellite morphology to realize multimodal imagingguided cancer radiotherapy function. ${ }^{40}$ The introduction of Se could greatly increase the conductivity and reduce the energy barrier between these three components, so that more electrons were transferred between the $\mathrm{Se}-\mathrm{Au}$ interface and the heterojunction at the $\mathrm{Au}-\mathrm{Fe}_{3} \mathrm{O}_{4}$ NPs interface. This synergistic interaction enhanced the energy transfer and facilitated more excited excitons generated by SeAuFe-EpC NPs, and thus promoting the transformation of ${ }^{3} \mathrm{O}_{2}$ to ${ }^{1} \mathrm{O}_{2}$, which ultimately led to the apoptosis of irreversible cancer cell. In addition, due

legumain to expose 1,2-thiol amino group of cysteine, where click cycloaddition further occurs with the contiguous cyano group of AuNPsDOX-CABT to form aggregates. (b) Living imaging of BALB/c mice after intravenous injection with AuNPs-Cy5.5-A\&C and control formulations for different time intervals; bar indicates radiant efficiency from $0.5 \times 10^{7}$ to $2.5 \times 10^{7}$. (c) PA images of AuNPs-A\&C incubated with legumain in the absence and presence of Ato acquired from phantom at $690 \mathrm{~nm}$, and AuNPs-PEG incubated with legumain; gray bars indicate ultrasound image of phantom, red bars indicate PA signal of phantom, and jet bars indicate the PA signal of AuNPs. Reproduced with permission from ref. 38, copyright: 2016, American Chemical Society. 
to the X-ray attenuation ability and high NIR absorption ability of AuNPs as well as the superparamagnetism of $\mathrm{Fe}_{3} \mathrm{O}_{4}$, SeAuFeEpC possessed a combined in vivo computer tomography, photoacoustic and magnetic resonance three-peak imaging capabilities to visually track nanoparticles. Cui group developed a multifunctional contrast agent-based gadolinium ionsmediated self-assembly of gold nanoclusters into monodisperse spherical nanoparticles (GNCNs). ${ }^{41}$ The as-prepared GNCNs could realize near-infrared fluorescence (NIRF) imaging, CT imaging and MR imaging. Liu group fabricated hpDNA-functionalized $\mathrm{NaYF}_{4} @ \mathrm{SiO}_{2}-\mathrm{Au}$ nanoconjugates base on the coupling of small-sized AuNPs $(\approx 2 \mathrm{~nm})$ and hairpin DNA (hpDNA) conjugation, which could realize simultaneous deeptissue imaging and site-specific administration of anticancer drugs (Fig. 6). ${ }^{42}$ In this system, upconversion nanoparticles were coated with silica and the hpDNA strands were anchored onto the AuNPs through Au-thiol bond. The coupling of Au to the upconversion nanoparticles could realize the modulation of luminescence in the NIR region $(\approx 800 \mathrm{~nm})$ for minimal attenuation in tissue, while the hpDNA strands allowed a high drug loading and releasing capacity. In vivo experiments showed that the tumor in the experimental group gradually decreased in comparison with the control groups. Therefore, the as-prepared nanoconjugates were promising probes for deep-tissue imaging and guided therapy. Li group synthesized a dumbbell-shaped heterogeneous copper selenide-gold nanocrystals (CSA) by simply mixing $\mathrm{Cu}_{2-x} \mathrm{Se}$ nanoparticles and $\mathrm{HAuCl}_{4}$ in an aqueous solution without using reducing agents. ${ }^{43}$ The obtained CSA composite exhibited significant enhancement of radiosensitization compared to $\mathrm{Cu}_{2-x} \mathrm{Se}$ nanocrystals (CS), AuNPs and their mixtures. In addition, the CSA composite possessed multi-modal imaging capacity including PAI, CT and
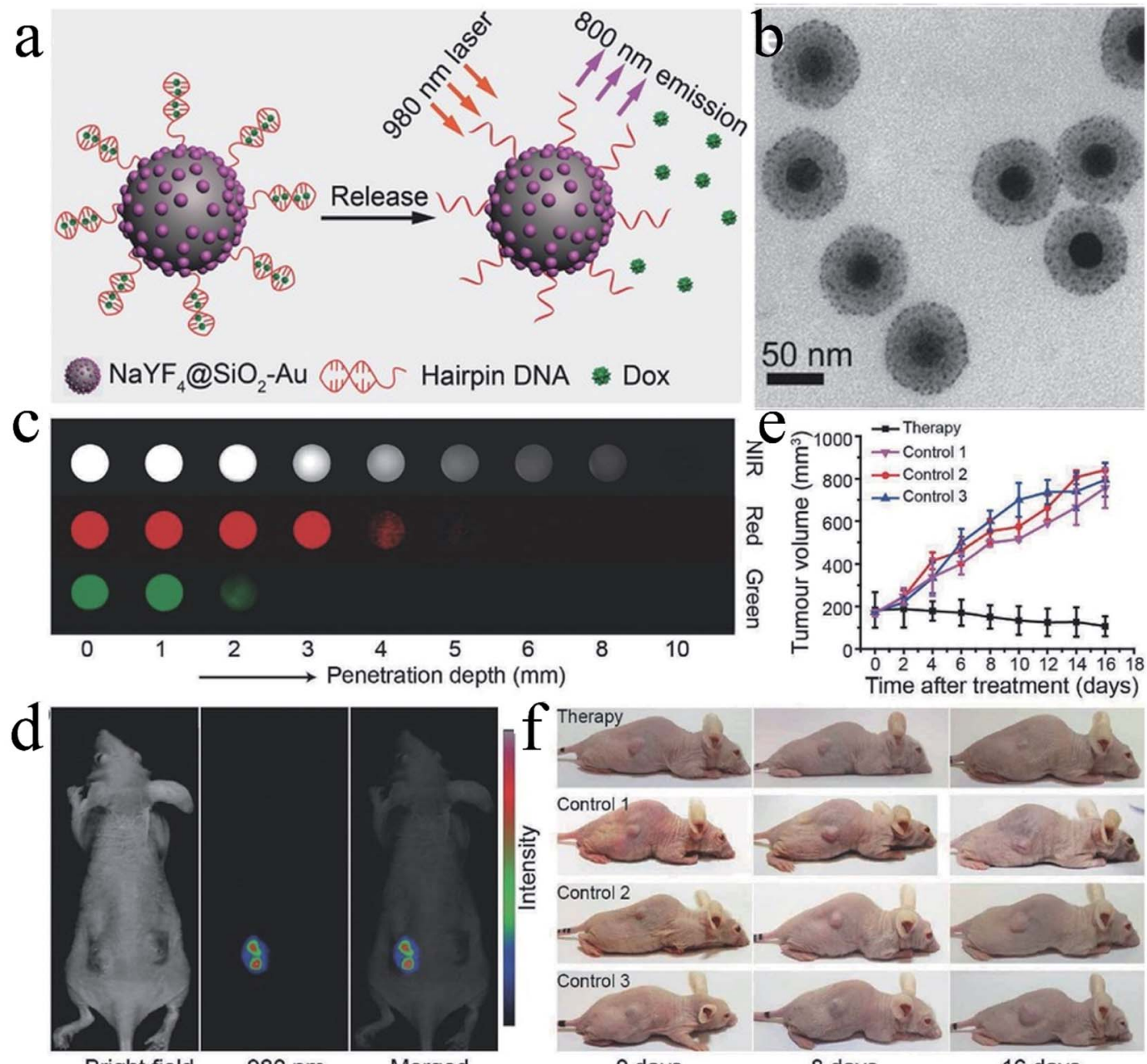

Fig. 6 (a) Schematic illustration of Dox drug release from hpDNA-modified NaYF4aSiO $\mathrm{C}_{2}-\mathrm{Au}$ nanoconjugates, triggered by a photon upconversion process. (b) TEM images of the $\mathrm{NaYF}_{4}\left(\mathrm{CSiO}_{2}-\mathrm{Au}\right.$ nanoparticles. (c) CCD imaging of NIR $(800 \mathrm{~nm})$, red $(660 \mathrm{~nm})$, and green (540 nm) emissions, recorded for the as-prepared $\mathrm{NaYF}_{4} \mathrm{aSiO}_{2}-\mathrm{Au}$ nanoconjugates and NaYF $4 \mathrm{Yb} / \mathrm{Er}(18: 2$ mol\%) aNaYF 4 nanocrystals, under pork muscle tissues of different depth. False-color images were taken under the $980 \mathrm{~nm}$ laser excitation. (d) In vivo upconversion imaging of the tumor-bearing mouse treated with the DNA-modified nanoconjugates. (e) Volumetric changes of the HeLa tumor, compiled as a function of duration time in four different modes of treatment. The tumor volume was normalized to their initial sizes. (f) The corresponding photographs of the tumor-bearing mice taken after various treatments. Reproduced with permission from ref. 42, copyright: 2017, Wiley. 
SPECT, as well as synergistic radiophotothermal therapy of cancer. These results illustrated that the CSA composite could realize the precise diagnosis and treatment through multimodal imaging guided radiophotothermal therapy. Zhu group synthesized a size tunable core-shell composite $\left(\mathrm{Au}-\mathrm{SiO}_{2}-\right.$ $\left.\mathrm{WO}_{3}\right){ }^{44}$ Briefly, a $\mathrm{WO}_{3}$ core with a diameter of $20-50 \mathrm{~nm}$ was coated with and intermediated silica layer $(10-60 \mathrm{~nm})$ followed by coating with Au shell (5-20 nm). Afterward, 4-mercaptobenzoic acid (4-MBA) was modified onto the surface of the Au shell and served as Raman reporters. The obtained $\mathrm{Au}-\mathrm{SiO}_{2}-$ $\mathrm{WO}_{3}$ composite exhibited high resolution Raman properties for single-molecule detection.

Metal-organic framework (MOF) are three-dimensional porous materials composed of inorganic metal nodes termed secondary building units linked together by rigid multitopic organic linkers. MOF possesses lots of advantages including structure diversity, large surface area, tunable pore size and functionalities, which are widely used in the area of storage, separation, sensing, catalysis and drug/gene delivery. ${ }^{45-50}$ MOF is firstly introduced into the area of drug delivery in 2006 by Férey group, subsequently, the MOF is widely used as vector for drug/gene delivery. ${ }^{51}$ Boyes group firstly fabricated a Gd MOF nanoparticles (GdMOF) followed by anchoring the AuNPs onto the surface of GdMOF. ${ }^{52}$ In this structure, GdMOF serves as MRI contrast agent, which possessed a high Gd payload, adjustable size and exhibited excellent capability of MR imaging. AuNPs were dispersed on the surface of the GdMOF structure and served as CT contrast agent. Therefore, integration of GdMOF with AuNPs together endowed this nanoplatform with the ability of MRI and CT imaging, which possessed significant attention in the early diagnosis of cancer. Lei group fabricated a core-shell structure with a peptide modified AuNPs core and a pH-sensitive MOF (ZIF-8) shell. ${ }^{53}$ This hybrid composite showed dual-recognition and could be used for monitoring the activity of lysosomal CaB. Briefly, ZIF-8 shell possesses excellent biocompatibility and exhibits highly activated response to the acidic environment. After disintegration of the ZIF-8 shell, the Au-peptide core exposed to the CaB and recognized by $\mathrm{Cab}$, thereby realizing a stepwise-responsive strategy for $\mathrm{CaB}$ detection. Triple-negative breast cancer (TNBC) is one of the most dangerous types of breast cancer with poor clinical outcome. Zeng group developed well defined AuNS@MOF-ZD2 nanocomposites by encapsulating a single AuNS within MIL-101- $\mathrm{NH}_{2}(\mathrm{Fe})$ to from core-shell structure to achieve specifically targeting toward triple-negative breast cancer (TNBC). ${ }^{54}$ Fig. 7B showed the morphologies of AuNS and AuNS@MOF by coating MOF with one to four cycles. The obtained core-shell AuNS@MOF-ZD2 composites exhibited remarkable $T_{1}$-weighted MR imaging and PTT toward TNBC with a photothermal conversion efficiency of $40.5 \%$. Meanwhile, the temperature in the tumor sites of targeted group was $5.5{ }^{\circ} \mathrm{C}$ higher than that of nontargeted group at $5 \mathrm{~min}$ postirradiation of $808 \mathrm{~nm}$ laser. In addition, the mice were alive well in the targeted group, while the mice were dead in the other control groups during the period of 40 days posttreatment. Zhang group reported a gold nanorod-based nanoplatform for the treatment of primary tumor and the inhibition of metastatic tumor through PTT. ${ }^{55}$ In these work, AuNR was coated with mesoporous silica (AuNR@MSN) followed by loading DOX. Thereafter, the AuNR@MSN-DOX was further coated with metal $\left(\mathrm{Gd}^{3+}\right)$-organic network (AuNR@MSN@MON). The MON membrane could offer superior strategy for inhibiting tumor metastasis, the Gd section endowed the NPs with the ability of MR imaging, the combination of PTT and chemotherapy obviously enhanced the antitumor therapeutic effects.

Every coin has two sides. In the ancient Rome, Janus, Rome God with two face, could look back on the past events and forward to the future. Janus NPs are composed of two different surface properties, which exhibiting absolutely different chemical or physical properties. Thereafter, Janus NPs have attracted significant attention in recent years due to its unique nature. Filice group reported a Janus nanoplatform consisted of $\mathrm{Fe}_{3} \mathrm{O}_{4}$ NPs/mesoporous silica core @ shell surface together with a gold nanoparticle surface. ${ }^{56}$ Afterward, cRGD and Dye were modified onto the Janus nanoplatform due to its anisotropy. Thereafter, the obtained Janus nanoplatform possessed multimodal imaging capacity including $\mathrm{MRI}\left(\mathrm{Fe}_{3} \mathrm{O}_{4}\right.$ core), CT (AuNP face) and fluorescent tracking (Dye), which could serve as a powerful nanoplatform for targeted cancer theranosis. Sonodynamic therapy (SDT) possesses high penetration, noninvasiveness and controllability, which is suitable for detection of deep-seated tumors. Hou group reported a monodisperse Au$\mathrm{Fe}_{2} \mathrm{C}$ Janus NPs with a diameter of $12 \mathrm{~nm} .{ }^{57}$ The experimental results illustrated that $\mathrm{Au}-\mathrm{Fe}_{2} \mathrm{C}$ Janus NPs could simultaneously realize $\mathrm{MR} / \mathrm{MSOT} / \mathrm{CT}$ imaging as well as photothermal therapy (30.2\%) under $808 \mathrm{~nm}$ laser irradiation.

Vesicle has a huge cavity, which can be used for drug loading. Different from the Janus NPs mentioned above, Yang group fabricated $\mathrm{Au}-\mathrm{MnO}$ Janus NPs followed by coating with PEG and a ROS-sensitive polymer, ${ }^{58}$ afterward, the obtained Janus nanocomposites self-assemble into a vesicle which could disassembled into small Au-MnO Janus NPs, subsequently, the MnO section degraded and release $\mathrm{Mn}^{2+}$ for chemodynamic therapy. This Janus NPs integrated photoacoustic imaging together with $T_{1}$-weighted MR imaging and realise synergistic SDT and CDT. A similar structure was also reported by Chen group (Fig. 8). ${ }^{59}$ They synthesized $\mathrm{Fe}_{3} \mathrm{O}_{4}-\mathrm{Au}$ Janus NPs followed by grafting PEG on the Au surface and ROS-generating poly(lipid hydroperoxide) (PLHP) on the $\mathrm{Fe}_{3} \mathrm{O}_{4}$ surface, respectively. Afterward, this multifunctional $\mathrm{Fe}_{3} \mathrm{O}_{4}-\mathrm{Au}$ Janus NPs assembled into bilayer vesicles and DOX was also loaded in the cavity of the vesicles. Therefore, this multifunctional nanoplatform could realize multimodal imaging including MRI, PA and PET. In addition, the acidic tumor environment could trigger the disassemble of vesicles into single Janus NPs, thereby enhancing local ROS generation due to intracellular biochemical reaction between PLHP and the released ferrous ion $\left(\mathrm{Fe}^{2+}\right)$. Combined ROS and DOX therapy, the tumors were obviously ablated in the mice treated with JNP@PLHPVP-PEG Ve-DOX compared to the control group, which was further demonstrated through MR imaging. 

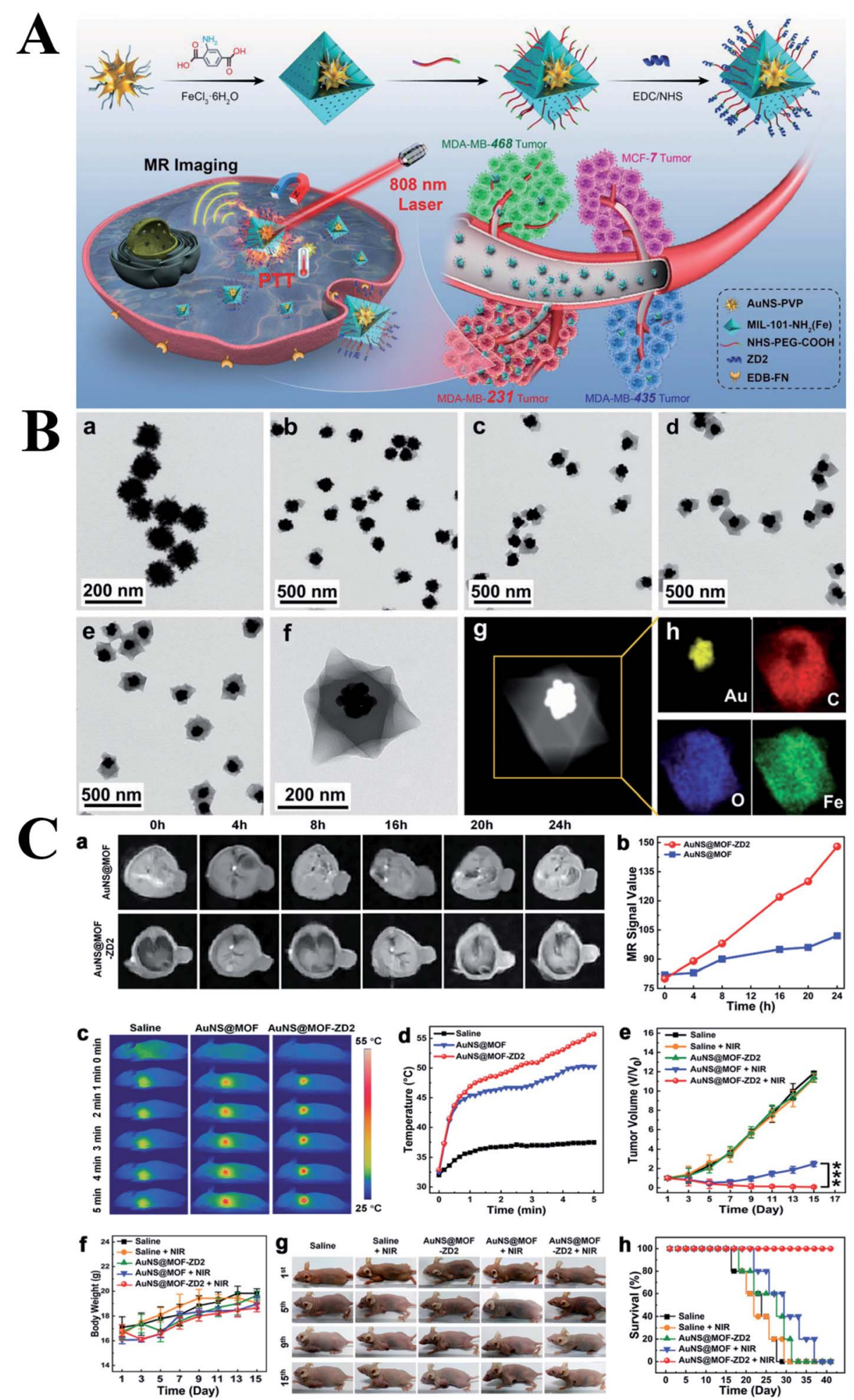

Fig. 7 (A) Schematic illustration of the synthesis of AUNS@MOF-ZD2 nanoprobes, and application for $T_{1}$-weighted MR imaging and photothermal therapy specifically toward MDA-MB-231 tumor (TNBC). (B) TEM images of (a) AuNS-PVP, (b-d) AuNS@MOF by coating MOF with one to three cycles, respectively. (e and f) TEM images of final AuNS@MOF by coating MOF with four cycles. (g) STEM image, and (h) the corresponding EDX elemental mapping of AuNS@MOF. (C) (a) $T_{1}$-weighted MR images and (b) the corresponding MR signal intensity of MDA-MB-231 tumorbearing nude mice, intravenously injected with AuNS@MOF and AuNS@MOF-ZD2 (200 $\mu \mathrm{L}, 2 \mathrm{mg} \mathrm{mL}^{-1}$ ), at different time point of postinjection. (c) NIR thermal images and (d) the corresponding temperature variation curves of MDA-MB-231 tumor-bearing mice, intravenously injected with saline, AuNS@MOF and AuNS@MOF-ZD2 (200 $\left.\mu \mathrm{L}, 2 \mathrm{mg} \mathrm{mL}^{-1}\right)$, after $24 \mathrm{~h}$ of postinjection ( $808 \mathrm{~nm}$ laser, $\left.1.2 \mathrm{~W} \mathrm{~cm}{ }^{-2}, 5 \mathrm{~min}\right)$. (e) Relative tumor volume curves $(* * * p<0.001)$, (f) body weight curves, $(\mathrm{g})$ the corresponding photographs, and (h) survival rate curves of MDA-MB-231 tumorbearing nude mice in different groups (five mice per group). Reproduced with permission from ref. 54, copyright: 2018, Wiley. 


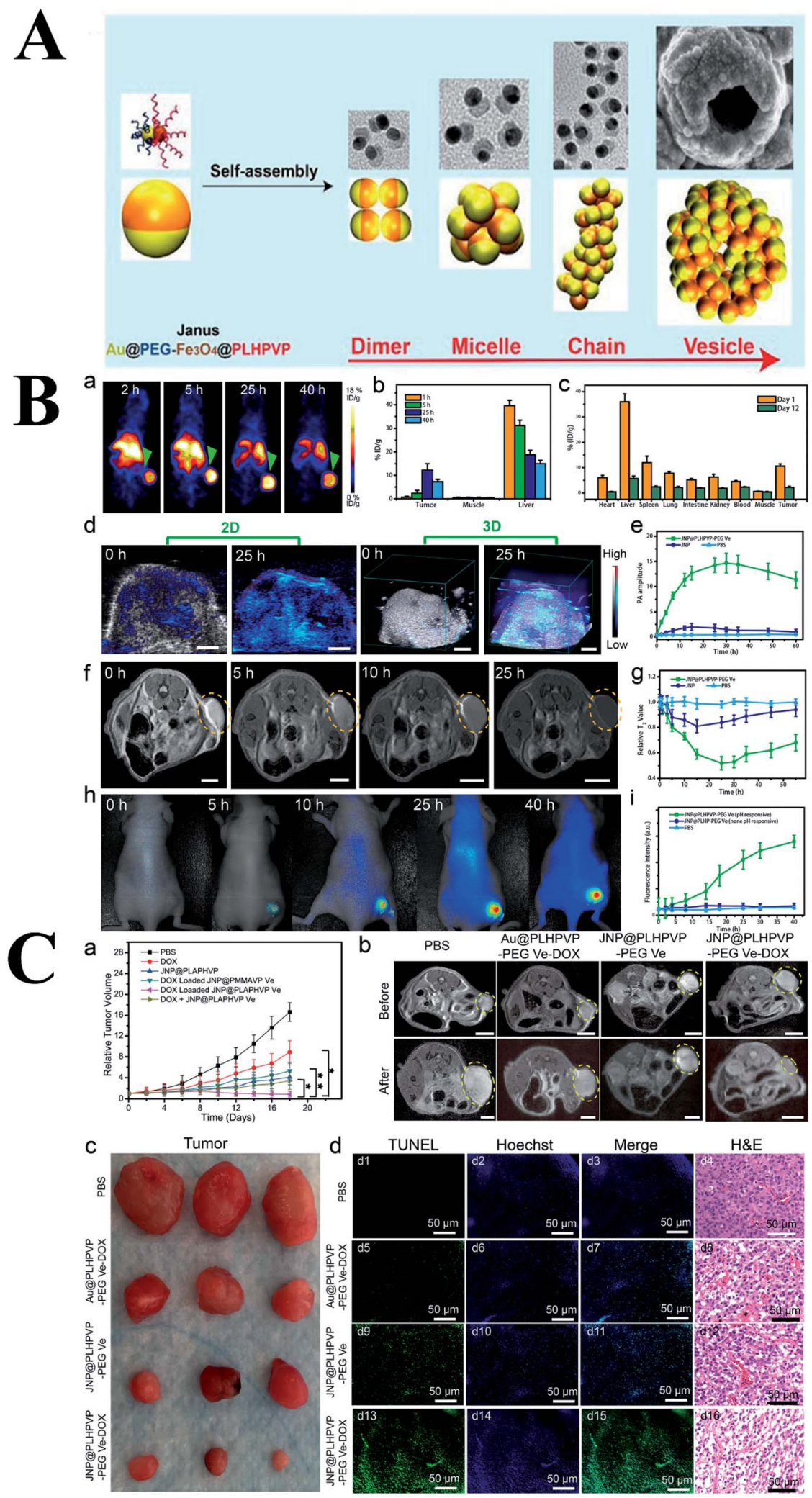

Fig. 8 (A) Schematic representation of the self-assembly of amphiphilic Janus NPs into dimer, micelle, chain, and bilayered vesicles and their corresponding simulation results. (B) In vivo imaging and biodistribution studies of the hybrid vesicles. (a) PET imaging of U87MG tumor-bearing mice at different time points after intravenous injection of $\left[{ }^{64} \mathrm{Cu}\right]$ radiolabeled JNP vesicles. (b) PET region of interest (ROI) analysis of tumor, muscle, and liver at 1, 5, 25, and $40 \mathrm{~h}$ postinjection. (c) Direct tissue sampling biodistribution in different organs at day 1 and 12 postinjection analyzed by ICP-MS. (d) In vivo PA images and (e) average PA intensity in the tumor after intravenous injection of the hybrid vesicles. (f) $T_{2}{ }^{-}$ weighted MR images and $(\mathrm{g})$ the relative $T_{2}$ values of the tumor before and after intravenous injection of vesicles at different times. Scale bar: 5 $\mathrm{mm}$. (h) In vivo fluorescence imaging and (i) tumor signal intensity after injection of IRDy800CW-loaded vesicles. (C) In vivo cancer therapy effect 


\section{Membrane-coated gold nanoparticles for imaging and therapy}

As early as 1965, bilayer phospholipid system, named liposomes, was first established in 1965. Liposomes are nano-sized to micro-sized vesicles consisted of a phospholipid bilayer and cavity. Subsequently, liposomes can be prepared according to different method with various composition, size, charge and structure. Various liposomes were designed as important vectors, including drug/gene loading, long-circulating liposomes, triggered release liposomes. ${ }^{60-64}$ In 1995 , Doxil $囚$, the first FDA-approved nano-drug is based on PEGylated nanoliposomes, which can passively targeted to tumor region due to the EPR effect, thereby releasing doxorubicin. ${ }^{65}$

Zhu group engineered a multifunctional nanoplatform based on gold nanostar @ $\mathrm{SiO}_{2} / \mathrm{DOX}$ encapsulated in liposome (GSMS@lip).66 This nanoplatform not only served as vector for delivering therapeutic drugs, but also had high photothermal efficiency. When the nanoplatform exposure to the nearinfrared (NIR) laser, the ambient temperature was raised due to the photothermal effect of gold nanostar, thereby rupturing the thermosensitive liposome and triggering the drug release. Benefiting from the synergistic effect of chemotherapeutic drugs and photothermal effect, the as-prepared multifunctional nanoplatform could effectively inhibit the tumor growth. Next, De group fabricated a biodegradable plasmon resonant liposome gold nanoparticle (LiposAu NP), which was capable of killing cancer cells through PTT (Fig. 9A). ${ }^{67}$ In this work, liposome was prepared through thin film hydration method followed by coating gold nanoparticles on the surface of liposome. The obtained LiposAu NPs were prepared to achieve a size range of $100-120 \mathrm{~nm}$. After exposing to $750 \mathrm{~nm}$ laser, the tumor xenograft was complete ablated, thereby degrading into small NPs and clearing through hepato-biliary and renal route. A similar work was also reported by Gao group, they designed and synthesized a gold nanoshell coated oleanolic acid liposome mediating by chitosan (GNOLs). ${ }^{68}$ This liposome exhibited a slow release of oleanolic acid at $\mathrm{pH} 7.4$ while a rapid release at $\mathrm{pH}$ 5.5, which is good for tumor targeting drug release. In addition, the gold nanoshell could activate photothermal effect and trigger drug release after NIR irradiation.

Undoubtedly, liposomes possess many advantages, but they also face some deficiencies, for example, liposomes can be cleared by reticuloendothelial system (RES) or mononuclear phagocytic system (MPS) due to surface charge or size. Thereafter, K. A. Dawson firstly proposed the concept of protein corona, who found that the NPs can absorb protein on the surface when they circled in the blood, thereby changing the charge and dispersion in the biological environment. Subsequently, various proteins were anchored onto the surface of NPs to endow the NPs with new biological properties, thereby improving their performance including cellular uptake, immune response, clearance and toxicity. Li group fabricated a theranostic nanoplatform via gadolinium (Gd)-based bovine serum albumin (BSA) hybrid-coated hollow gold nanoshells (Au@BSA-Gd). ${ }^{69}$ In addition, phototherapeutic agent [indocyanine green (ICG)] was loading into the cavity of Au NPs. Therefore, this Au NPs could be developed as theranostic nanoplatform for NIRF/PA/CT/MR quadmodal imaging as well as precise cancer therapy. Briefly, hollow Au nanoshells were fabricated by the silica-template removal method. Afterward, BSA-Gd complex was anchored onto the surface of Au nanoshells through EDC chemistry followed by loading of ICG. The in vivo results demonstrated that Au@BSA-Gd could realize in vivo quadmodal NIRF/PA/CT/MR imaging capability as well as combined PTT and PDT therapy. Gold nanocages (AuNCs) and gold nanoclusters (AuClusters) are two classes of nanostructures with excellent attractive properties (Fig. 9B). ${ }^{70} \mathrm{Wu}$ group integrated AuNCs and AuClusters into one theranostic nanoplatform for fluorescent and optoacoustic bimodal imaging as well as PTT. In this work, gold nanocages (AuNCs) were synthesized using a galvanic replacement between silver nanocubes and chloroauric acid $\left(\mathrm{HAuCl}_{4}\right)$. Next, Erlotinib (EB) was loading into the AuNCs followed by modification of PLL. Finally, Au cluster@BSA was anchored onto the surface of AuNCs via electrostatic interaction. The obtained hybrid gold could release EB in the presence of lysosomal proteases and low $\mathrm{pH}$ and induce fluorescent restoration for imaging. As shown in Fig. 9B-e, no signal could be observed in the control group, while distinct signals could be observed from the AuNC in tumor, liver, spleen and lungs. In addition, the drug release rate would further accelerate under NIR irradiation, and the results demonstrated that the combined therapeutic efficacy could effectively inhibit the tumor growth.

However, chemical crosslinking usually causes protein denaturation and greatly limit their applications. Reb blood cells (RBCs) are natural carrier in the human body, and become a hotspot in drug delivery research own to its non-immunogenic and biocompatible characters. Troyer group engineered RBCs as a carrier for loading AuNPs, thereby acting as an efficient CTcontrast agent (Fig. 10A). ${ }^{71}$ In this work, AuNPs $(5 \mathrm{~nm})$ were anchored onto the surface of RBCs through the lipid thiol ligand without altering its shape, size and surface properties. The average size of RBCs is about 7 micron, which greatly inhibit their applications in vivo, but providing a new concept for construction of RBCs-based vector. Subsequently, Zhang group camouflaged AuNPs with red blood cell membranes to endow these Au NPs with the surface properties of RBCs, which could be less susceptible to clearance by phagocytic cells (Fig. 10B). ${ }^{72}$ Briefly, RBC membrane coating technique was applied onto the inorganic AuNPs via extruder repeatedly according to the previous work. The size of obtained RBC

of JNP vesicles. (a) Tumor growth profiles in mice after different treatments ( $* P<0.05 ; * * P 0.01)$. (b) Tumor MR images before and 18 days after treatment with different formulations. (c) Representative photographs of the dissected tumors in different treatment groups. (d) TUNEL-labeled tumor sections treated with different formulations (green color: positive TUNEL staining, blue color: nuclei stained by Hoechst 33342) and histological analysis of tumor sections after staining with H\&E in different treatment groups. Reproduced with permission from ref. 59, copyright: 2019, ACS. 

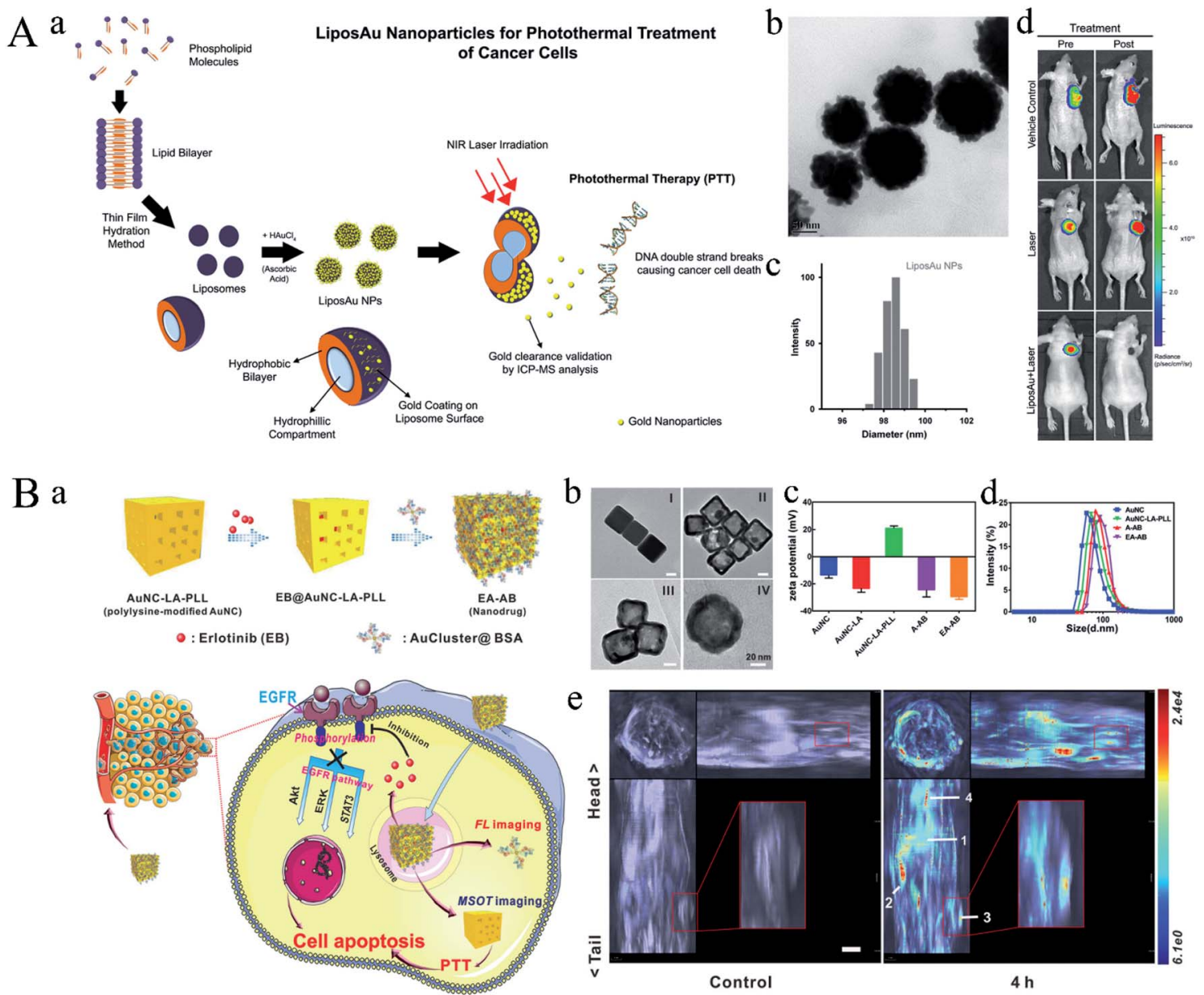

Fig. 9 (A) (a) Schematic diagram representing the principle of synthesis of LiposAu NPs and their mode of action to perform photothermal treatment causing intracellular DNA damage. (b) TEM image of LiposAu NPs. (Scale $=50 \mathrm{~nm}$ ). (c) DLS size distribution of LiposAu NPs. (d) Representative pre- and post-treatment in vivo bioluminescence images of mice bearing HT1080-fluc2-turboFP tumor xenografts. Reproduced with permission from ref. 67, copyright: 2015, American Chemical Society. (B) Schematic illustration for (a) fabrication of EA-AB and its imaging capability and dual actions on tumor cell. (b) TEM images of (I) Ag nanocube, (II) AuNC, (III) AuNC-LA-PLL, and (IV) EA-AB. Scale bar: 20 nm. (c) Zeta potential of AuNC, AuNC-LA, AuNC-LA-PLL, A-AB (AuNC-AuCluster@BSA without loading EB), and EA-AB. Columns represent mean \pm standard deviation (SD), $n=3$. (d) Diameter distribution of AuNC, AuNC-LA-PLL, A-AB, and EA-AB as determined by dynamic light scattering. (e) In vivo orthogonal 3D MSOT view of 4T1 tumor-bearing mice before and after tail vein injection of the EA-AB dispersion (AuNC content: 80 mg $\left.\mathrm{kg}^{-1}\right) . n=3$ per group. Reproduced with permission from ref. 70, copyright: 2019, Wiley.

membrane-camouflaged AuNPs slightly increased (from 70.1 to 86.5 ) while the zeta potential increased from -42.2 to -35.1 . In addition, a thiolated fluorescent probe and an antibody bind assay further demonstrated that the AuNPs were successfully camouflaged by RBC membrane. Similarly, Yang group fabricated RBC-membrane-coated gold nanocages (AuNCs), which exhibited excellent colloidal stability. ${ }^{73}$ The results indicated that the fusion of RBC membrane did not change the porous and hollow structure of AuNCs, but significantly enhanced the in vivo blood retention and circulation lifetime. In addition, van Hest group constructed a Janus polymeric motors based on gold nanoparticles and chitosan/heparin followed by coated with erythrocyte membrane, which could be used for photothermal therapy and successfully applied in thrombus ablation. ${ }^{74}$ In this work, chitosan (a positive charge polysaccharide, $\mathrm{CHI}$ ) and heparin (a negative glycosaminoglycan, Hep) were selected to construct biodegradable capsule with a gold layer through sputter coating, thereby obtaining a NIR-responsive Janus structure. Experiment results illustrated that the change of temperature could lead to the movement of the EM-JPMs through the self-thermophoresis effect after exposing to laser irradiation. This biohybrid EM-JPMs exhibited excellent performance in thrombus ablation.

\section{Outlook and challenges}

Over the past two decades, various gold-based nanomaterials with different shape and size were prepared, and the development of gold-based NPs dramatically attracts scientists' attention in the medical fields such as imaging, gene/drug delivery and therapy. These multifunctional gold nanomaterials were widely developed as imaging-guided in vivo cancer therapy. Nevertheless, there are also many challenges in this field: (1) biocompatibility, and (2) commercialization of AuNPs. 

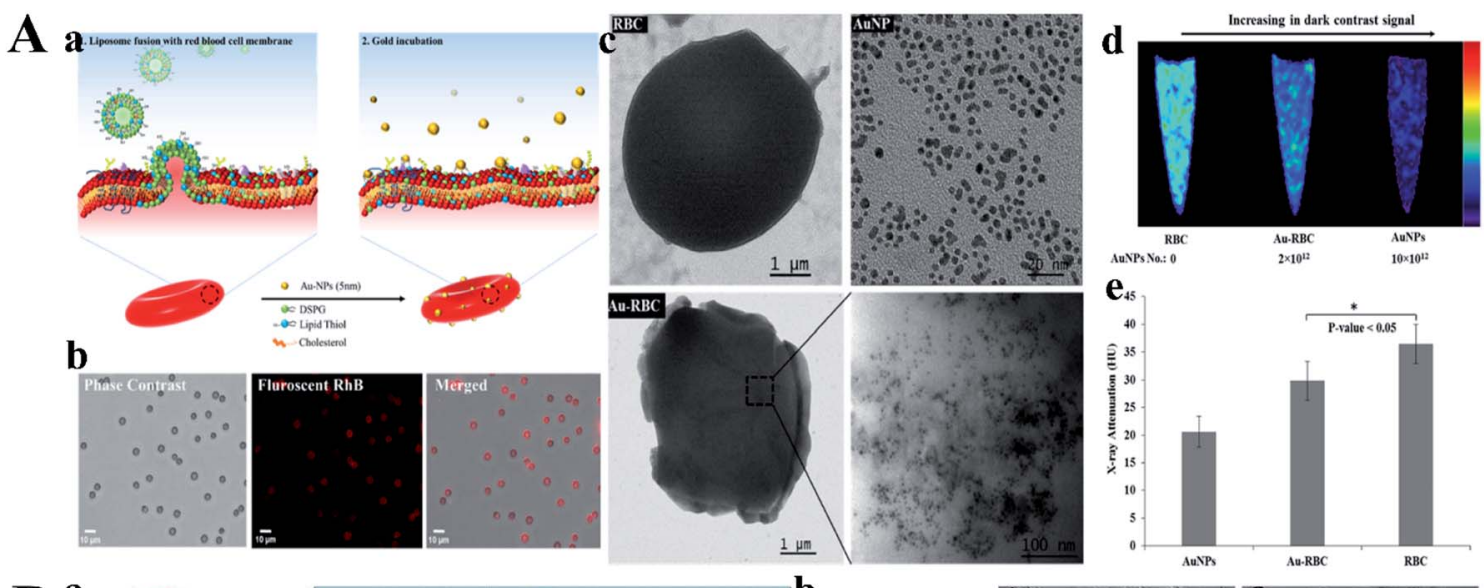

$\mathbf{B}^{\mathbf{a}}$
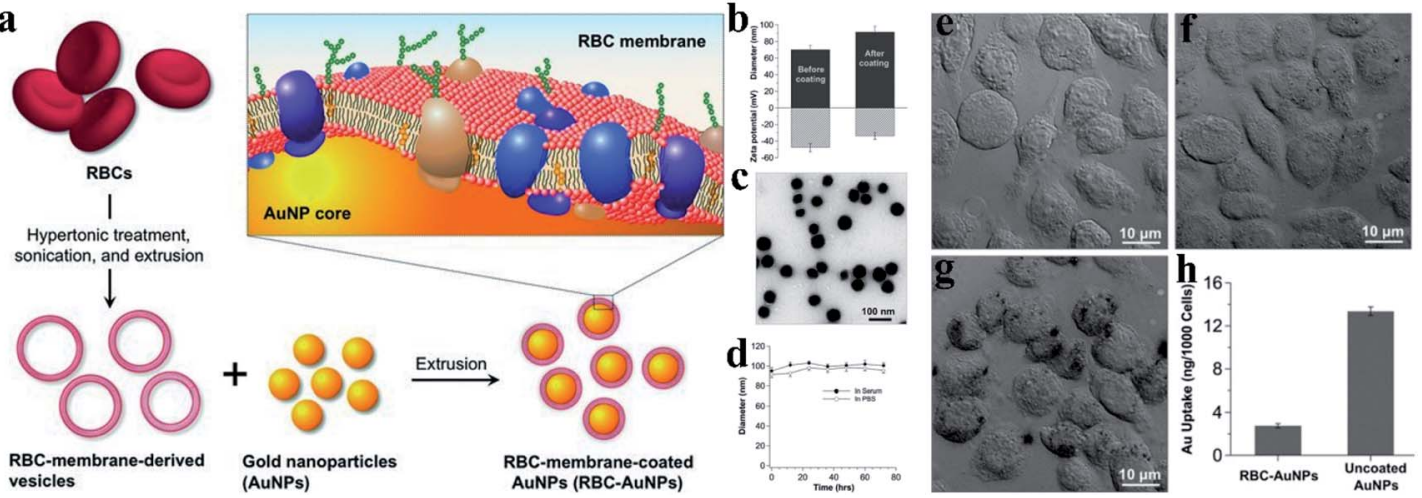

Fig. 10 (A) (a) Strategic fusion mechanism showing gold nanoplating. (b) Microscopic demonstration of RhB-labeled fusogenic liposome fused RBC, phase contrast, fluorescent, and merged images. (c) TEM images of RBC, AuNP, and Au-RBC. Inset of Au-RBC electron micrographs of Au-RBC shows the plating of RBC surface with clusters of $5 \mathrm{~nm}$ AuNPs. (d) CT images of pure RBC, Au-RBC, and AuNPs embedded in agarose gel. (e) X-ray attenuation (Housefield Unit, HU) of AuNPs, Au-RBC, RBC ( $* P<0.05$, student's $t$-test). Reproduced with permission from ref. 71, copyright: 2017, American Chemical Society. (B) (a) Schematic illustration of the preparation process of AuNPs functionalized with RBC membranes (RBC-AuNPs). (b) Hydrodynamic size (diameter, $\mathrm{nm}$ ) and surface zeta potential $(\mathrm{mV})$ of AuNPs before and after RBC membrane coating measured by dynamic light scattering (DLS). (c) A representative TEM image showing the spherical core-shell structure of the RBC-AuNPs under negative staining with uranyl acetate. (d) Stability of the resulting RBC-AuNPs in 100\% serum and 1× PBS, respectively, by monitoring particle size (diameter, nm) over a span of $72 \mathrm{~h}$. (e-g) RBC membrane coating inhibits macrophage uptake. Bright field microscopic images of J774 murine macrophage cells incubated for $30 \mathrm{~min}$ in culture medium (e), $25 \mu \mathrm{g} \mathrm{mL}{ }^{-1} \mathrm{RBC}$-AuNPs (f), and $25 \mu \mathrm{g} \mathrm{mL}^{-1}$ uncoated AuNPs (g). (h) Quantitative analysis of macrophage uptake of RBC-AuNPs and uncoated AuNPs determined by ICP-MS measurements. Reproduced with permission from ref. 72, copyright: 2013, Wiley

\subsection{Biocompatibility}

There is no doubt that gold-based nanoparticles possess lots of excellent properties, but, they still face some problems. Biocompatibility is the first priority, especially the long-term cytotoxicity. The cytotoxicity of AuNPs depends on various parameters, including size, cell type, surface coating, tissue distribution and so on, among which size is the most important parameter. ${ }^{75-77}$ The previous literature demonstrated that small AuNPs (4-5 nm) possesses higher toxicity than that of large AuNPs (about $20 \mathrm{~nm}$ ). ${ }^{78}$ Another literature reported that positively charged gold-dendrimer complex with a size of $5 \mathrm{~nm}$ were excreted after 5 days, while negatively/neutrally charged AuNPs with a size of $5 \mathrm{~nm}$ or AuNPs with a size above $11 \mathrm{~nm}$ showed much lower excretion, only about $10 \%$ of the initial dose was excreted. ${ }^{79,80}$ However, a simple chemical modification of AuNPs will greatly increase the cellular uptake efficiency and reduce the toxicity, such as PEG coating. ${ }^{81}$ Jeong group found that PEG-coated AuNPs with a diameter of $13 \mathrm{~nm}$ induced acute inflammation and apoptosis in human liver, ${ }^{82}$ while Kreyling group found that PEG-coated AuNPs were mostly accumulated in the liver and spleen. ${ }^{83}$

\subsection{Commercialization of AuNPs}

Despite AuNPs-based theranostic nanoplatforms have ushered a new period of vigorous development, few commercial opportunities have been created for their utilization in clinic. One major obstacle is the cost. For example, Xia group reported that $200 \mu \mathrm{L}$ of PEGylated AuNPs (Au atom mass $\approx 0.8 \mathrm{mg}$ per mouse) was used for in vivo photothermal treatment of mice $(\sim 20 \mathrm{~g})$, thus, the cost of PEGylated AuNPs for a normal human is very high. ${ }^{84}$ Therefore, these problems cannot be ignored, especially in the clinical application, which have become a pressing issue placed in front of modern scientist.

\section{Conclusion}

In this review, we summarize the fabrication and application of gold-based nanoparticles in recent decades. Initially, various 
polymers are anchored onto the surface of gold and thereby endowing the AuNPs with excellent stability as well as various properties in vivo. Next, gold-based hybrid NPs are fabricated to further expanded their properties and applications. Finally, membrane-mediated AuNPs (liposome or RBC membrane) are developed to improve the recognition ability and circulation time in blood, and thereby improving diagnosis and treatment effects. All these techniques greatly broaden the applications of gold in vitro and in vivo. Consequently, various literature report that a great many gold-based theranostic platform are developed, which focus on the animal modes, but nearly none of them show significant potential for cancer therapy in clinic. Therefore, scientists from various disciplines should make great efforts to cooperate in order to overcome the barriers, including chemistry, material, biology and engineering.

\section{Conflicts of interest}

There are no conflicts to declare.

\section{Acknowledgements}

This work was supported by the Science and Technology Foundation of Wuhan (2020020601012326), Graduate Innovative Fund of Wuhan Institute of Technology (CX2019026), Research Project of Hubei Provincial Department of Education of China (D20191504), Scientific Research Foundation of Wuhan Institute of Technology (K201914).

\section{Notes and references}

1 R. L. Siegel, K. D. Miller, H. E. Fuchs and A. Jemal, Ca-Cancer J. Clin., 2022, 72, 7-33.

2 R. L. Siegel, K. D. Miller and A. Jemal, Ca-Cancer J. Clin., 2020, 70, 7-30.

3 J. A. Webb and R. Bardhan, Nanoscale, 2014, 6, 2502-2530.

4 Y. Xia, W. Li, C. M. Cobley, J. Chen, X. Xia, Q. Zhang, M. Yang, E. C. Cho and P. K. Brown, Acc. Chem. Res., 2011, 44, 914-924.

5 J. Guo, K. Rahme, Y. He, L. L. Li, J. D. Holmes and C. M. O'Driscoll, Int. J. Nanomed., 2017, 12, 6131-6152.

6 M. Sharifi, F. Attar, A. A. Saboury, K. Akhtari, N. Hooshmand, A. Hasan, M. A. El-Sayed and M. Falahati, J. Controlled Release, 2019, 311-312, 170-189.

7 S. K. Libutti, G. F. Paciotti, A. A. Byrnes, H. R. Alexander, W. E. Gannon, M. Walker, G. D. Seidel, N. Yuldasheva and L. Tamarkin, Clin. Cancer Res., 2010, 16, 6139.

8 A. N. Kharlamov, A. E. Tyurnina, V. S. Veselova, O. P. Kovtun, V. Y. Shur and J. L. Gabinsky, Nanoscale, 2015, 7, 8003-8015.

9 M. P. Melancon, M. Zhou and C. Li, Acc. Chem. Res., 2011, 44, 947-956.

10 W. Mao, Y. J. Son and H. S. Yoo, Nanoscale, 2020, 12, 1499615020.

11 J. Stone, S. Jackson and D. Wright, WIREs Nanomedicine and Nanobiotechnology, 2011, 3, 100-109.

12 L. Vigderman, B. P. Khanal and E. R. Zubarev, Adv. Mater., 2012, 24, 4811-4841.
13 N. Kaur, R. N. Aditya, A. Singh and T.-R. Kuo, Nanoscale Res. Lett., 2018, 13, 302.

14 L.-Y. Chen, C.-W. Wang, Z. Yuan and H.-T. Chang, Anal. Chem., 2015, 87, 216-229.

15 E. Porret, X. Le Guével and J.-L. Coll, J. Mater. Chem. B, 2020, 8, 2216-2232.

16 Y. Zheng, J. Wu, H. Jiang and X. Wang, Coord. Chem. Rev., 2021, 431, 213689.

17 V. Alimardani, G. Farahavar, S. Salehi, S. Taghizadeh, M. R. Ghiasi and S. S. Abolmaali, Front. Mater. Sci., 2021, 15, 494-511.

18 S. Hwang, J. Nam, S. Jung, J. Song, H. Doh and S. Kim, Nanomedicine, 2014, 9, 2003-2022.

19 P. Deveci, J. Inclusion Phenom. Macrocyclic Chem., 2021, 99, 23-31.

20 G. Frens, Nat. Phys. Sci., 1973, 241, 20-22.

21 J. Turkevich, P. C. Stevenson and J. Hillier, Discuss. Faraday Soc., 1951, 11, 55-75.

22 A. Gole and C. J. Murphy, Chem. Mater., 2004, 16, 3633-3640. 23 S. E. Skrabalak, L. Au, X. Li and Y. Xia, Nat. Protoc., 2007, 2, 2182-2190.

24 R. J. Holbrook, N. Rammohan, M. W. Rotz, K. W. MacRenaris, A. T. Preslar and T. J. Meade, Nano Lett., 2016, 16, 3202-3209.

25 Y. Yang, L. Zhang, J. Cai, X. Li, D. Cheng, H. Su, J. Zhang, S. Liu, H. Shi, Y. Zhang and C. Zhang, ACS Appl. Nano Mater., 2016, 8, 1718-1732.

26 W.-H. Chen, Q. Lei, G.-F. Luo, H.-Z. Jia, S. Hong, Y.-X. Liu, Y.-J. Cheng and X.-Z. Zhang, ACS Appl. Nano Mater., 2015, 7, 17171-17180.

27 Y. Zhao, Y. Huang, H. Zhu, Q. Zhu and Y. Xia, J. Am. Chem. Soc., 2016, 138, 16645-16654.

28 H. Liu, Y. Xu, S. Wen, J. Zhu, L. Zheng, M. Shen, J. Zhao, G. Zhang and X. Shi, Polym. Chem., 2013, 4, 1788-1795.

29 X. Li, K. Takeda, E. Yuba, A. Harada and K. Kono, J. Mater. Chem. B, 2014, 2, 4167-4176.

30 X. Li, M. Takashima, E. Yuba, A. Harada and K. Kono, Biomaterials, 2014, 35, 6576-6584.

31 F. J. Nicholls, M. W. Rotz, H. Ghuman, K. W. MacRenaris, T. J. Meade and M. Modo, Biomaterials, 2016, 77, 291-306.

32 E. R. Figueroa, A. Y. Lin, J. Yan, L. Luo, A. E. Foster and R. A. Drezek, Biomaterials, 2014, 35, 1725-1734.

33 H. Bao, Y. Xia, C. Yu, X. Ning, X. Liu, H. Fu, Z. Chen, J. Huang and Z. Zhang, Small, 2019, 15, 1904314.

34 G. Zhou, H. Xiao, X. Li, Y. Huang, W. Song, L. Song, M. Chen, D. Cheng and X. Shuai, Acta Biomater., 2017, 64, 223-236.

35 P. K. Kundu, D. Samanta, R. Leizrowice, B. Margulis, H. Zhao, M. Börner, T. Udayabhaskararao, D. Manna and R. Klajn, Nat. Chem., 2015, 7, 646-652.

36 X. Cheng, R. Sun, L. Yin, Z. Chai, H. Shi and M. Gao, Adv. Mater., 2017, 29, 1604894.

37 X. Gao, Q. Yue, Z. Liu, M. Ke, X. Zhou, S. Li, J. Zhang, R. Zhang, L. Chen, Y. Mao and C. Li, Adv. Mater., 2017, 29, 1603917.

38 S. Ruan, C. Hu, X. Tang, X. Cun, W. Xiao, K. Shi, Q. He and H. Gao, ACS Nano, 2016, 10, 10086-10098.

39 S. Rajkumar and M. Prabaharan, Colloids Surf., B, 2020, 186, 110701. 
40 H. Liu, W. Lin, L. He and T. Chen, Biomaterials, 2020, 226, 119545.

41 W. Hou, F. Xia, G. Alfranca, H. Yan, X. Zhi, Y. Liu, C. Peng, C. Zhang, J. M. de la Fuente and D. Cui, Biomaterials, 2017, 120, 103-114.

42 S. Han, A. Samanta, X. Xie, L. Huang, J. Peng, S. J. Park, D. B. L. Teh, Y. Choi, Y.-T. Chang, A. H. All, Y. Yang, B. Xing and X. Liu, Adv. Mater., 2017, 29, 1700244.

43 Q. Huang, S. Zhang, H. Zhang, Y. Han, H. Liu, F. Ren, Q. Sun, Z. Li and M. Gao, ACS Nano, 2019, 13, 1342-1353.

44 P. Martinez Pancorbo, K. Thummavichai, L. Clark, T. A. Tabish, J. Mansfield, B. Gardner, H. Chang, N. Stone and Y. Zhu, Adv. Funct. Mater., 2019, 29, 1903549.

45 O. K. Farha, A. Özgür Yazaydın, I. Eryazici, C. D. Malliakas, B. G. Hauser, M. G. Kanatzidis, S. T. Nguyen, R. Q. Snurr and J. T. Hupp, Nat. Chem., 2010, 2, 944-948.

46 P. Horcajada, T. Chalati, C. Serre, B. Gillet, C. Sebrie, T. Baati, J. F. Eubank, D. Heurtaux, P. Clayette, C. Kreuz, J.-S. Chang, Y. K. Hwang, V. Marsaud, P.-N. Bories, L. Cynober, S. Gil, G. Férey, P. Couvreur and R. Gref, Nat. Mater., 2010, 9, 172-178.

47 A. C. McKinlay, R. E. Morris, P. Horcajada, G. Férey, R. Gref, P. Couvreur and C. Serre, Angew. Chem., Int. Ed., 2010, 49, 6260-6266.

48 P. Horcajada, R. Gref, T. Baati, P. K. Allan, G. Maurin, P. Couvreur, G. Férey, R. E. Morris and C. Serre, Chem. Rev., 2012, 112, 1232-1268.

49 H. Zheng, Y. Zhang, L. Liu, W. Wan, P. Guo, A. M. Nyström and X. Zou, J. Am. Chem. Soc., 2016, 138, 962-968.

50 M. Kalaj, K. C. Bentz, S. Ayala, J. M. Palomba, K. S. Barcus, Y. Katayama and S. M. Cohen, Chem. Rev., 2020, 120, 8267-8302.

51 P. Horcajada, C. Serre, M. Vallet-Regí, M. Sebban, F. Taulelle and G. Férey, Angew. Chem., Int. Ed., 2006, 45, 5974-5978.

52 C. Tian, L. Zhu, F. Lin and S. G. Boyes, ACS Appl. Nano Mater., 2015, 7, 17765-17775.

53 H. Shen, J. Liu, J. Lei and H. Ju, Chem. Commun., 2018, 54, 9155-9158.

54 L. Zhang, C. Liu, Y. Gao, Z. Li, J. Xing, W. Ren, L. Zhang, A. Li, G. Lu, A. Wu and L. Zeng, Adv. Healthcare Mater., 2018, 7, 1801144.

55 J.-X. Fan, D.-W. Zheng, W.-W. Mei, S. Chen, S.-Y. Chen, S.-X. Cheng and X.-Z. Zhang, Small, 2017, 13, 1702714.

56 A. Sánchez, K. Ovejero Paredes, J. Ruiz-Cabello, P. MartínezRuíz, J. M. Pingarrón, R. Villalonga and M. Filice, ACS Appl. Nano Mater., 2018, 10, 31032-31043.

57 Y. Ju, H. Zhang, J. Yu, S. Tong, N. Tian, Z. Wang, X. Wang, X. Su, X. Chu, J. Lin, Y. Ding, G. Li, F. Sheng and Y. Hou, ACS Nano, 2017, 11, 9239-9248.

58 X. Lin, S. Liu, X. Zhang, R. Zhu, S. Chen, X. Chen, J. Song and H. Yang, Angew. Chem., Int. Ed., 2020, 59, 1682-1688.

59 J. Song, L. Lin, Z. Yang, R. Zhu, Z. Zhou, Z.-W. Li, F. Wang, J. Chen, H. Yang and X. Chen, J. Am. Chem. Soc., 2019, 141, 8158-8170.

60 A. Akbarzadeh, R. Rezaei-Sadabady, S. Davaran, S. W. Joo, N. Zarghami, Y. Hanifehpour, M. Samiei, M. Kouhi and K. Nejati-Koshki, Nanoscale Res. Lett., 2013, 8, 102.
61 T. M. Allen and P. R. Cullis, Adv. Drug Delivery Rev., 2013, 65, 36-48.

62 C. Chen, D. Han, C. Cai and X. Tang, J. Controlled Release, 2010, 142, 299-311.

63 H. Lv, S. Zhang, B. Wang, S. Cui and J. Yan, J. Controlled Release, 2006, 114, 100-109.

64 K. Maruyama, Adv. Drug Delivery Rev., 2011, 63, 161-169.

65 Y. Barenholz, J. Controlled Release, 2012, 160, 117-134.

66 Z. Cai, Y. Zhang, Z. He, L.-P. Jiang and J.-J. Zhu, ACS Appl. Bio Mater., 2020, 3, 5322-5330.

67 A. K. Rengan, A. B. Bukhari, A. Pradhan, R. Malhotra, R. Banerjee, R. Srivastava and A. De, Nano Lett., 2015, 15, 842-848.

68 L. Luo, Y. Bian, Y. Liu, X. Zhang, M. Wang, S. Xing, L. Li and D. Gao, Small, 2016, 12, 4103-4112.

69 Q. You, Q. Sun, M. Yu, J. Wang, S. Wang, L. Liu, Y. Cheng, Y. Wang, Y. Song, F. Tan and N. Li, ACS Appl. Nano Mater., 2017, 9, 40017-40030.

70 C. Zhan, Y. Huang, G. Lin, S. Huang, F. Zeng and S. Wu, Small, 2019, 15, 1900309.

71 S. Aryal, T. D. T. Nguyen, A. Pitchaimani, T. B. Shrestha, D. Biller and D. Troyer, ACS Biomater. Sci. Eng., 2017, 3, 36-41.

72 W. Gao, C.-M. J. Hu, R. H. Fang, B. T. Luk, J. Su and L. Zhang, Adv. Mater., 2013, 25, 3549-3553.

73 J.-G. Piao, L. Wang, F. Gao, Y.-Z. You, Y. Xiong and L. Yang, ACS Nano, 2014, 8, 10414-10425.

74 J. Shao, M. Abdelghani, G. Shen, S. Cao, D. S. Williams and J. C. M. van Hest, ACS Nano, 2018, 12, 4877-4885.

75 N. Li, P. Zhao and D. Astruc, Angew. Chem., Int. Ed., 2014, 53, 1756-1789.

76 I. Fratoddi, I. Venditti, C. Cametti and M. V. Russo, Nano Res., 2015, 8, 1771-1799.

77 A. M. Alkilany, P. K. Nagaria, C. R. Hexel, T. J. Shaw, C. J. Murphy and M. D. Wyatt, Small, 2009, 5, 701-708.

78 X. D. Zhang, D. Wu, X. Shen, P. X. Liu, N. Yang, B. Zhao, H. Zhang, Y. M. Sun, L. A. Zhang and F. Y. Fan, Int. J. Nanomed., 2011, 6, 2071-2081.

79 L. Balogh, S. S. Nigavekar, B. M. Nair, W. Lesnia, C. Zhang, L. Y. Sung, M. S. T. Kariapper, A. El-Jawahri, M. Llanes, B. Bolton, F. Mamou, W. Tan, A. Hutson, L. Minc and M. K. Khan, Nanomedicine, 2007, 3, 281-296.

80 J. B. Vines, J. H. Yoon, N. E. Ryu, D. J. Lim and H. Park, Front. Chem., 2019, 7, 167.

81 J. A. Zamora-Justo, P. Abrica-González, G. R. VázquezMartínez, A. Muñoz-Diosdado, J. A. Balderas-López and M. Ibáñez-Hernández, J. Nanomater., 2019, 2019, 5982047.

82 W.-S. Cho, M. Cho, J. Jeong, M. Choi, H.-Y. Cho, B. S. Han, S. H. Kim, H. O. Kim, Y. T. Lim, B. H. Chung and J. Jeong, Toxicol. Appl. Pharmacol., 2009, 236, 16-24.

83 J. Lipka, M. Semmler-Behnke, R. A. Sperling, A. Wenk, S. Takenaka, C. Schleh, T. Kissel, W. J. Parak and W. G. Kreyling, Biomaterials, 2010, 31, 6574-6581.

84 Y. Wang, K. C. L. Black, H. Luehmann, W. Li, Y. Zhang, X. Cai, D. Wan, S.-Y. Liu, M. Li, P. Kim, Z.-Y. Li, L. V. Wang, Y. Liu and Y. Xia, ACS Nano, 2013, 7, 2068-2077. 